J. DIFFERENTIAL GEOMETRY

52 (1999) $73-115$

\title{
DISCRIMINANT OF THETA DIVISORS AND QUILLEN METRICS
}

\author{
KEN-ICHI YOSHIKAWA
}

\begin{abstract}
We show that analytic torsion of smooth theta divisor is represented by a Siegel modular form characterizing the Andreotti-Mayer locus.
\end{abstract}

\section{Introduction}

In the theory of modular forms of one variable, the unique cusp form of weight 12 called Jacobi's $\Delta$-function:

$$
\Delta(\tau)=q \prod_{n=1}^{\infty}\left(1-q^{n}\right)^{24}, \quad q=\exp (2 \pi i \tau)
$$

is one of the most important objects. There are several view points to see it. From an algebraic view point, it is the discriminant of elliptic curves. To be precise, let $E_{\tau}:=\mathbb{C} / \mathbb{Z} \oplus \mathbb{Z} \tau(\tau \in \mathbb{H})$ be an elliptic curve and take its Weierstrass model: $y^{2}=4 x^{3}-g_{2}(\tau) x-g_{3}(\tau)$. Jacobi discovered the following formula:

$$
g_{2}(\tau)^{3}-27 g_{3}(\tau)^{2}=(2 \pi)^{12} \Delta(\tau)
$$

Namely $\Delta(\tau)$ is the discriminant of the polynomial $4 x^{3}-g_{2}(\tau) x-g_{3}(\tau)$.

From an analytic view point, $\Delta(\tau)$ is essentially the Ray-Singer analytic torsion. Equipped with the Kähler metric $g_{\tau}=(\operatorname{Im} \tau)^{-1}|d z|^{2}$, analytic torsion of (the trivial line bundle on) $E_{\tau}$ is, by definition (Definition 2.1), $\tau\left(E_{\tau}\right)=\exp \left(\zeta_{\tau}^{\prime}(0)\right)$ where

$$
\zeta_{\tau}(s)=(2 \pi)^{-2 s} \sum_{(m, n) \neq(0,0)} \frac{(\operatorname{Im} \tau)^{s}}{|m+n \tau|^{2 s}}
$$

Received April 13, 1998, and, in revised form, February 8, 1999. 
is the $\zeta$-function of Laplacian. Then, Kronecker's first limit formula yields

$$
\tau\left(E_{\tau}\right)=(2 \pi)^{2}\|\Delta(\tau)\|^{-\frac{1}{6}} .
$$

Here, $\|f(\tau)\|^{2}:=(\operatorname{Im} \tau)^{k}|f(\tau)|^{2}$ is the Peteresson norm. A naive consideration expects that analytic torsion of an Abelian variety might imply a higher dimensional analogue of Jacobi's $\Delta$-function. Unfortunately, it is not the case. In fact, Ray-Singer ([25]) showed that analytic torsion of an Abelian variety of dimension $\geq 2$ equipped with any flat Kähler metric is 1 .

The purpose of this article is to show that analytic torsion of the theta divisor is represented by a Siegel modular form analogous to Jacobi's $\Delta$-function.

Let $\mathfrak{S}_{g}$ be the Siegel upper half space of genus $g>1$. Let $\Lambda_{\tau} \subset \mathbb{C}^{g}$ be the lattice defined by

$$
\Lambda_{\tau}:=\mathbb{Z}_{1} e_{1} \oplus \cdots \oplus \mathbb{Z} e_{g} \oplus \mathbb{Z}_{\tau_{1}} \oplus \cdots \oplus \mathbb{Z}_{\boldsymbol{g}}
$$

where $1_{g}=\left(e_{1}, \cdots, e_{g}\right)$ and

$$
\tau=\left(\tau_{1}, \cdots, \tau_{g}\right) \in \mathfrak{S}_{g}\left(e_{i}, \tau_{j} \in \mathbb{C}^{g}\right) .
$$

Let $A_{\tau}=\mathbb{C}^{g} / \Lambda_{\tau}$ be an Abelian variety, and

$$
\Theta_{\tau}:=\left\{z \in A_{\tau} ; \theta(z, \tau)=0\right\}
$$

its theta divisor where

$$
\theta(z, \tau):=\sum_{m \in \mathbb{Z}^{g}} \exp \left(\pi i^{t} m \tau m+2 \pi i^{t} m z\right)
$$

is the theta function. Let $N_{g}:=\left\{\tau \in \mathfrak{S}_{g}\right.$; Sing $\left.\Theta_{\tau} \neq \emptyset\right\}$ be the discriminant locus of theta divisors called Andreotti-Mayer locus. Let $g_{\tau}:={ }^{t} d z(\operatorname{Im} \tau)^{-1} d \bar{z}$ be the flat invariant Kähler metric of $A_{\tau}$ and $g_{\Theta_{\tau}}:=\left.g_{\tau}\right|_{\Theta_{\tau}}$ its induced Kähler metric on $\Theta_{\tau}$.

Main Theorem (Theorem 5.2). Suppose that $g>1$ and $\Theta_{\tau}$ is smooth. Then, $\tau\left(\Theta_{\tau}\right)$, the analytic torsion of $\left(\Theta_{\tau}, g_{\Theta_{\tau}}\right)$, is represented by

$$
\tau\left(\Theta_{\tau}\right)=\left\|\Delta_{g}(\tau)\right\|^{\frac{(-1)^{g+1} 2}{(g+1) !}}
$$


where $\Delta_{g}(\tau)$ is a Siegel cusp form of weight $\frac{(g+3) \cdot g !}{2}$ with zero divisor $N_{g}$ (and with character when $g=2$ ) vanishing at the highest dimensional cusp of order $\frac{(g+1) !}{12}$, and

$$
\left\|\Delta_{g}(\tau)\right\|^{2}:=(\operatorname{det} \operatorname{Im} \tau)^{\frac{(g+3) \cdot g !}{2}}\left|\Delta_{g}(\tau)\right|^{2}
$$

its Petersson norm.

According to Debarre ([9]), $N_{g}$ consists of two irreducible components $\theta_{\text {null,g }}$ and $N_{g}^{\prime}$ considered as a divisor on the modular variety $S p(2 g ; \mathbb{Z}) \backslash \mathfrak{S}_{g}$, which implies that $\chi_{g}(\tau)$, the product of all even theta constants, is a divisor of $\Delta_{g}(\tau)$ as in the case of Jacobi's $\Delta$ function. Namely, there exists $J_{g}(\tau)$, a Siegel modular form of weight $\frac{(g+3) \cdot g !}{4}-2^{g-3}\left(2^{g}+1\right)$ with zero divisor $N_{g}^{\prime}$, such that

$$
\Delta_{g}(\tau)=\chi_{g}(\tau) J_{g}(\tau)^{2}
$$

Since $J_{g}(\tau)=C_{g}$ is a constant for $g=2,3$, and $J_{4}(\tau)$ is the Schottky form which characterizes the Jacobian locus in $\mathfrak{S}_{4}$, we know $\Delta_{g}(\tau)$ explicitly (up to some universal constant) in terms of theta constants for $g<5$. (For a formula for $J_{4}(\tau)$, see [16].) We remark that the result in Main Theorem was essentially known in the case $g=2$ ([6], [27]). For any smooth ample divisor on a polarized Abelian variety, its analytic torsion is treated in section 5 and 6 in terms of Quillen metrics as a generalized version of Main Theorem. Roughly speaking, one can compute the Quillen metric via the defining equation of the projective dual variety of Abelian varieties relative to the given polarization (Theorems $5.1,6.1,6.3)$. Although only the principally polarized case is treated there, we remark that the same arguments works for arbitrarily polarized case. As an example, we discuss the case of $|2 \Theta|$ for Abelian surfaces in section 7 where the equation of Kummer's quartic surface appears.

A very interesting problem of finding the field of definition of $\Delta_{g}(\tau)$ was raised to the author by the referee and several other people. Unfortunately, he could not find any answer and leave it to the reader. (See Conjecture 6.1.) $\Delta(\tau)$ and $\Delta_{2}(\tau)$ are eigenfunctions for the Hecke operators. Thus, at least as a working hypothesis, it looks worth asking if so is $\Delta_{g}(\tau)$ when $g \geq 3$.

After finishing the first version of this paper, he knew that Jorgenson and Kramer treat related subjects by using Green currents ([17], [18]). 


\section{Acknowledgement}

This work was initiated while the author was staying at the Fourier Institut. He is grateful to its hospitality. He thanks professors O. Debarre, J.-P. Demailly, R. Lazarsfeld and C. Mourougane for answering his questions. He also thanks the referee and professors H. Gillet and C. Soulé for their valuable advices on the earlier version of this article which improved section 7 , inspired section 6 and corrected many mistakes. Finally, his thanks are to professor S. Mukai. Through several discussions with him, the author could learn much about the subject.

\section{Determinant bundles and Quillen metrics}

In this section, we recall some properties of Quillen metrics which will be used later. For the general treatment of Quillen metrics, see [2], [26], [11].

Let $\pi: X \rightarrow S$ be a proper smooth morphism of Kähler manifolds. The determinant bundle $\lambda\left(\mathcal{O}_{X}\right)$ is defined by the following formula:

$$
\lambda\left(\mathcal{O}_{X}\right):=\bigotimes_{q \geq 0}\left(\operatorname{det} R^{q} \pi_{*} \mathcal{O}_{X}\right)^{(-1)^{q}}
$$

Let $g_{X / S}$ be a Kähler metric on the relative tangent bundle. Namely, it is a Hermitian metric on $T X / S:=\operatorname{ker} \pi_{*}$ such that $\left.g_{X / S}\right|_{X_{t}}$ is Kähler for any fiber $X_{t}:=\pi^{-1}(t)$. By the Hodge theory, identify $\lambda\left(\mathcal{O}_{X}\right)_{t}$ with the determinant of harmonic forms:

$$
\begin{aligned}
\lambda\left(\mathcal{O}_{X}\right)_{t} & =\bigotimes_{q \geq 0}\left(\bigwedge^{\max } H^{q}\left(X_{t}, \mathcal{O}_{X_{t}}\right)\right)^{(-1)^{q}} \\
& \cong \bigotimes_{q \geq 0}\left(\bigwedge^{\max } \mathcal{H}^{0, q}\left(X_{t}\right)\right)^{(-1)^{q}}
\end{aligned}
$$

where $\mathcal{H}^{0, q}\left(X_{t}\right)$ stands for the harmonic $(0, q)$-forms. Since $\mathcal{H}^{0, q}\left(X_{t}\right)$ carries the natural Hermitian structure by the integration of harmonic forms, so does $\lambda\left(\mathcal{O}_{X}\right)_{t}$ via the identification (2.2). This metric is called the $L^{2}$-metric of $\lambda\left(\mathcal{O}_{X}\right)$ relative to $g_{X / S}$ and is denoted by $\|\cdot\|_{L^{2}}$.

Let $\square_{t}^{0, q}$ be the $\bar{\partial}$-Laplacian acting on $(0, q)$-forms on $X_{t}$, and $\zeta_{t}^{0, q}(s)$ its spectral zeta function. It is well known that $\zeta_{t}^{0, q}(s)$ extends to a meromorphic function on the whole complex plane and is regular at $s=0$. 
Definition 2.1. The Quillen metric of $\lambda\left(\mathcal{O}_{X}\right)$ relative to $g_{X / S}$ is defined by

$$
\|\cdot\|_{Q}^{2}(t):=\tau\left(X_{t}\right)\|\cdot\|_{L^{2}}^{2}(t),
$$

where $\tau\left(X_{t}\right)$ is the Ray-Singer analytic torsion:

$$
\tau\left(X_{t}\right):=\prod_{q \geq 0}\left(\operatorname{det} \square_{t}^{0, q}\right)^{(-1)^{q} q}, \quad \operatorname{det} \square_{t}^{0, q}:=\exp \left(-\left.\frac{d}{d s}\right|_{s=0} \zeta_{t}^{0, q}(s)\right) .
$$

It is known that $\|\cdot\|_{Q}$ is a smooth Hermitian metric on $\lambda\left(\mathcal{O}_{X}\right)$ if the morphism is smooth. For smooth Kähler morphisms, the curvature and anomaly formulas for the Quillen metrics are computed by BismutGillet-Soulé.

Theorem 2.1 ([3]). The curvature form of $\|\cdot\|_{Q}$ is given by

$$
c_{1}\left(\lambda\left(\mathcal{O}_{X}\right),\|\cdot\|_{Q}\right)=\pi_{*}\left(\operatorname{Td}\left(T X / S, g_{X / S}\right)\right)^{(1,1)}
$$

where $\alpha^{(p, p)}$ stands for the $(p, p)$-part of the form $\alpha$.

Theorem 2.2 ([3]). Let $g_{X / S}, g_{X / S}^{\prime}$ be Kähler metrics of $T X / S$, and $\|\cdot\|_{Q},\|\cdot\|_{Q}^{\prime}$ be the Quillen metrics of $\lambda\left(\mathcal{O}_{X}\right)$ relative to $g_{X / S}, g_{X / S}^{\prime}$ respectively. Then,

$$
\log \left(\frac{\|\cdot\|_{Q}^{\prime}}{\|\cdot\|_{Q}}\right)^{2}=\pi_{*}\left(\widetilde{\mathrm{Td}}\left(T X / S ; g_{X / S}, g_{X / S}^{\prime}\right)\right)^{(0,0)}
$$

where $\widetilde{\mathrm{Td}}\left(T X / S ; g_{X / S}, g_{X / S}^{\prime}\right)$ is the Bott-Chern secondary class of $T X / S$ relative to the Todd form and $g_{X / S}, g_{X / S}^{\prime}$.

Consider the case that the morphism is not smooth. Let $S$ be the unit disc and $\pi: X \rightarrow S$ be a proper surjective holomorphic function. $(\pi, X, S)$ is said to be a smoothing of IHS if $\pi$ is of maximal rank outside of finite number of points in $X_{0}$. In particular, $X_{0}$ has only isolated hypersurface singularities (IHS) and $X_{t}$ is smooth for any $t \neq 0$.

Theorem 2.3 ([28]). Let $(\pi, X, S)$ be a smoothing of IHS which is projective over $S$. Let $g_{X}$ be a Kähler metric of $X$, and $g_{X / S}$ the induced metric on $T X / S$. Then, $\|\cdot\|_{Q}$ is a singular Hermitian metric whose curvature current is

$$
c_{1}\left(\lambda\left(\mathcal{O}_{X}\right),\|\cdot\|_{Q}\right)=\frac{(-1)^{n+1}}{(n+2) !} \mu\left(\operatorname{Sing} X_{0}\right) \delta_{0}+\pi_{*}\left(\operatorname{Td}\left(T X / S, g_{X / S}\right)\right)^{(1,1)}
$$


where $n=\operatorname{dim}_{\mathbb{C}} X / S$, $\delta_{0}$ the Dirac measure supported at $0, \mu\left(\operatorname{Sing} X_{0}\right)$ the total Milnor number, and $\pi_{*}\left(\operatorname{Td}\left(T X / S, g_{X / S}\right)\right)^{(1,1)} \in L_{l o c}^{p}(S)$ for some $p>1$.

We also need Bismut-Lebeau's theorem. (For the general setting, see [4].)

Theorem 2.4 ([4]). Let $X$ be a compact Kähler manifold and $\left(Y, g_{Y}=\left.g_{X}\right|_{Y}\right)$ its smooth hypersurface with induced metric. Let $L=$ $[Y]$ be the line bundle defined by $Y$ and $s_{Y}$ its canonical section, i.e., $\left(s_{Y}\right)_{0}=[Y]$. Let $h_{L}=\|\cdot\|_{L}^{2}$ be a Hermitian metric of $L$, and $g_{N_{Y / X}}$ a Hermitian metric of $N_{Y / X}$ such that it holds on $Y,\left\|d s_{Y}\right\|_{N_{Y / X}^{*} \otimes L_{Y}}^{2} \equiv 1$, where $L_{Y}:=\left.L\right|_{Y}$ and $d s_{Y} \in H^{0}\left(Y, N_{Y / X}^{*} \otimes L\right)$. Let $\lambda_{X}\left(L^{-1}\right), \lambda_{X}$ and $\lambda_{Y}$ be the determinant of cohomologies equipped with the Quillen metrics relative to $g_{X}, g_{Y}$ and $h_{L^{-1}}$. Let $\sigma$ be the canonical element of $\lambda:=\lambda_{Y} \otimes \lambda_{X}^{-1} \otimes \lambda_{X}\left(L^{-1}\right)$. Then,

$$
\begin{aligned}
\log \|\sigma\|_{Q}^{2}= & -\int_{X} \operatorname{Td}\left(T X, g_{X}\right) \operatorname{Td}^{-1}\left(L, h_{L}\right) \log \|s\|_{L}^{2} \\
& +\int_{Y} \operatorname{Td}^{-1}\left(N_{Y / X}, g_{N_{Y / X}}\right) \widehat{\mathrm{Td}}(\overline{\mathcal{E}}) \\
& -\int_{X} \operatorname{Td}(T X) R(T X)+\int_{Y} \operatorname{Td}(T Y) R(T Y),
\end{aligned}
$$

where $R$ is the Gillet-Soulé genus, and $\widetilde{\mathrm{Td}}(\overline{\mathcal{E}})$ is the Bott-Chern class relative to the Todd genus and the exact sequence of the following Hermitian vector bundles

$$
\overline{\mathcal{E}}: 0 \rightarrow\left(T Y, g_{Y}\right) \rightarrow\left(\left.T X\right|_{Y},\left.g_{X}\right|_{Y}\right) \rightarrow\left(N_{Y / X}, g_{N_{Y / X}}\right) \rightarrow 0 .
$$

Since we treat Abelian varieties later, let us summarize the analytic torsion of certain line bundles over an Abelian variety. Let $A$ be an Abelian variety of dimension $g, \omega$ a flat Kähler metric, and $(L, h)$ an ample Hermitian line bundle whose Chern form is $\omega$. We denote by $\tau\left(A, L^{m}, \omega\right)$ the analytic torsion of $\left(L^{m}, h^{\otimes m}\right)$ relative to the metric $\omega$.

Proposition 2.1 ([5], [25]).

$$
\log \tau\left(A, L^{m}, \omega\right)= \begin{cases}\frac{1}{2} \rho\left(L^{m}\right) \log \frac{\rho\left(L^{m}\right)}{(2 \pi)^{g} \rho(\omega)} & (m>0), \\ 0 & (m=0), \\ (-1)^{g+1} \frac{1}{2} \rho\left(L^{-m}\right) \log \frac{\rho\left(L^{-m}\right)}{(2 \pi)^{g} \rho(\omega)} & (m<0),\end{cases}
$$


where $\rho(F):=c_{1}(F)^{g} / g$ ! for a line bundle and $\rho(\omega)=\operatorname{vol}(A, \omega)=$ $\int_{A} \omega^{g} / g !$

Proof. The case $m>0$ follows from [5, Proposition 4.2], and the case $m=0$ from [25]. Thus, it is enough to show the case $m<0$. Put $m=-n$ and $n>0$. To compute $\tau\left(A_{\tau}, L_{\tau}^{-n}\right)$, let

$$
*: \wedge^{0, q}\left(L^{-1}\right) \rightarrow \wedge^{g, g-q}(L)
$$

be the Hodge $*$-operator. Since $*$-operator commutes with the Laplacian; $* \square_{L^{-1}}^{0, q} \phi=\square_{L}^{g, g-q} * \phi,\left(\forall \phi \in \wedge^{0, q}\left(L^{-n}\right)\right), \square_{L^{-n}}^{0, q}$ and $\square_{L^{n}}^{g, g-q}$ have the same spectrum. Thus, the spectral zeta functions $\zeta^{0, q}\left(s, L^{-n}\right)$ of $\square_{L^{-n}}^{0, q}$ and $\zeta^{g, g-q}\left(s, L^{n}\right)$ of $\square_{L^{n}}^{g, g-q}$ coincide. As the canonical bundle of $A_{\tau}$ is trivial and is flat equipped with $\omega$, we find

$$
\zeta^{0, q}\left(s, L^{-n}\right)=\zeta^{g, g-q}\left(s, L^{n}\right)=\zeta^{0, g-q}\left(s, L^{n}\right),
$$

which, combined with [5, Proposition 4.2], yields

$$
\begin{aligned}
\log \tau\left(A_{\tau}, L^{-n}, \omega\right) & =\left.\sum_{q=0}^{g}(-1)^{q+1} q \frac{d}{d s}\right|_{s=0} \zeta^{0, g-q}\left(s, L^{n}\right) \\
& =\left.\sum_{q=0}^{g}(-1)^{q}(g-q) \frac{d}{d s}\right|_{s=0} \zeta^{0, g-q}\left(s, L^{n}\right) \\
& =(-1)^{g+1} \tau\left(A, L^{n}, \omega\right) \\
& =(-1)^{g+1} \frac{1}{2} \rho\left(L^{n}\right) \log \frac{\rho\left(L^{n}\right)}{(2 \pi)^{g} \rho(\omega)}
\end{aligned}
$$

where we have used $\sum_{q}(-1)^{q} \zeta^{0, q}\left(s, L^{n}\right) \equiv 0$ in the second equality.

q.e.d.

\section{Theta functions}

In this section, we collect fundamental facts about the theta function and the Siegel modular group without proofs. Details are found in [15], [23], [22] and [20].

Let $\mathfrak{S}_{g}$ be the Siegel upper half space of genus $g$. Let $\Lambda \subset \mathbb{C}^{g} \times \mathfrak{S}_{g}$ be a family of lattices in $\mathbb{C}^{g}$ defined by

$$
\Lambda_{\tau}:=\mathbb{Z} e_{1} \oplus \cdots \oplus \mathbb{Z} e_{g} \oplus \mathbb{Z} \tau_{1} \oplus \cdots \oplus \mathbb{Z} \tau_{g},
$$


where $1_{g}=\left(e_{1}, \cdots, e_{g}\right)$ and $\tau=\left(\tau_{1}, \cdots, \tau_{g}\right) \in \mathfrak{S}_{g}$. Let

$$
p: \mathbb{A}:=\mathbb{C}^{g} \times \mathfrak{S}_{g} / \Lambda \rightarrow \mathfrak{S}_{g}
$$

be the universal family of principally polarized Abelian varieties over $\mathfrak{S}_{g}$ whose fiber at $\tau$ is $A_{\tau}=\mathbb{C}^{g} / \Lambda_{\tau}$.

For any $m \geq 1$, we define a line bundle on $\mathbb{A}$ denoted by $L_{m}\left(=L_{1}^{\otimes m}\right)$; a function $f$ on $\mathbb{C}^{g}$ is a section of $L_{m, \tau}$ if and only if, for any $k, l \in \mathbb{Z}^{g}$,

$$
f(z+k+\tau l)=\exp \left(-\pi \sqrt{-1} m^{t} l \tau l-2 \pi \sqrt{-1} m^{t} l z\right) f(z) .
$$

When $m=1$, we write $L:=L_{1}$. Put $B_{m}=m^{-1} \mathbb{Z}^{g} / \mathbb{Z}^{g}$. For $a, b \in \mathbb{R}^{g}$, let

$$
\begin{array}{r}
\theta_{a, b}(z, \tau)=\sum_{n \in \mathbb{Z}^{g}} \exp \left(\pi \sqrt{-1}^{t}(n+a) \tau(n+a)\right. \\
\left.+2 \pi \sqrt{-1}^{t}(n+a)(z+b)\right)
\end{array}
$$

be the theta function. For any $a \in B_{m}$, put $\theta_{a}(\tau)=\theta_{a}:=\theta_{a, 0}(m z, m \tau)$.

Proposition 3.1 ([15, Chap.II], [20, Chap.5], [23, I, Chap.II]). For any $a \in B_{m}, \theta_{a} \in H^{0}\left(\mathfrak{S}_{g}, p_{*} L_{m}\right)$ and there exists a trivialization as $\mathcal{O}_{\mathfrak{S}_{g}}$-module:

$$
p_{*} L_{m}=\bigoplus_{a \in B_{m}} \mathcal{O}_{\mathfrak{S}_{g}} \theta_{a}
$$

Put $\theta(z, \tau):=\theta_{0,0}(z, \tau)$. Let $p: \Theta:=\{(z, \tau) \in \mathbb{A} ; \theta(z, \tau)=0\} \rightarrow \mathfrak{S}_{g}$ be the universal family of theta divisors. Then, $L$ is the line bundle defined by the divisor $\Theta$. Let $\Gamma_{g}=S p(2 g ; \mathbb{Z})$ be the integral symplectic group acting on $\mathbb{A}$ as follows:

$$
\gamma \cdot(z, \tau)=\left({ }^{t}(C \tau+D)^{-1} z,(A \tau+B)(C \tau+D)^{-1}\right), \quad \gamma=\left(\begin{array}{ll}
A & B \\
C & D
\end{array}\right)
$$

It is known that not every element of $\Gamma_{g}$ preserves $L$. Following Igusa, define

$$
\Gamma_{g}(1,2):=\left\{\left(\begin{array}{cc}
A & B \\
C & D
\end{array}\right) \in \Gamma_{g} ;\left({ }^{t} A C\right)_{0} \equiv\left({ }^{t} B D\right)_{0} \equiv 0 \quad \bmod 2\right\}
$$

where $X_{0}=\left(x_{i j} \delta_{i j}\right)$ denotes the diagonal for $X=\left(x_{i j}\right) \in M(g, \mathbb{Z})$. 
Proposition 3.2 ([15, Chap.II], [20, Chap.8]). There exists an unitary representation $\rho_{m}: \Gamma_{g}(1,2) \rightarrow U\left(\mathbb{C}^{m^{g}}\right)=U\left(V_{m}\right)$ such that, for any $\gamma \in \Gamma_{g}(1,2)$,

$$
\begin{aligned}
& \theta_{a, 0}(m \gamma \cdot z, m \gamma \cdot \tau) \\
& \quad=j(\tau, \gamma)^{\frac{1}{2}} \exp \left(\pi \sqrt{-1}^{t} z(C \tau+D)^{-1} C z\right) \sum_{b \in B_{m}} u_{a b}(\gamma) \theta_{b, 0}(m z, m \tau),
\end{aligned}
$$

where $\rho_{m}(\gamma)=\left(u_{a b}(\gamma)\right)_{a, b \in B_{m}}$ and $j(\tau, \gamma)=\operatorname{det}(C \tau+D)$. In particular, $\Gamma_{g}(1,2)$ preserves $L_{m}$ for any $m$.

Define a Hermitian metric $h_{L}$ on $L$ by

$$
\begin{aligned}
\|\theta\|_{L}^{2}(z, \tau) & =h_{L}(\theta, \theta)(z, \tau) \\
& :=|\theta(z, \tau)|^{2} \exp \left(-2 \pi^{t} \operatorname{Im} z(\operatorname{Im} \tau)^{-1} \operatorname{Im} z\right)
\end{aligned}
$$

and also by $h_{L_{m}}:=h_{L}^{\otimes m}$ on $L_{m}$. Then, $h_{L}$ is a natural metric in the sense that

$$
c_{1}\left(L, h_{L}\right)=g_{\tau}=\frac{\sqrt{-1}}{2} d z(\operatorname{Im} \tau)^{-1} d \bar{z}
$$

where the Kähler metric $g_{\tau}$ is identified with its Kähler form. With respect to $h_{L_{m}}$ and $g_{\tau}$, the length of $\left\{\theta_{a}\right\}_{a \in B_{m}}$ is given by the following formula ([15, Chap.II Lemma 7$],[20, \S 4.3$, pp.35, §5.4])

$$
\left(\theta_{a}(\tau), \theta_{b}(\tau)\right)_{L^{2}}=\{\operatorname{det}(2 m \operatorname{Im} \tau)\}^{-\frac{1}{2}} \delta_{a b} .
$$

Remark. Our $\theta_{a}$ is different from Kempf's $\eta_{\mathcal{L}}\left(\delta_{a}\right)(z)$ ([20, pp.41 $\left.\left(^{*}\right)\right]$ ). To obtain the norm of $\theta_{a}$, we must replace $\tau$ to $m \tau$ and choose $\tilde{e}=m 1_{g}$ in $[20$, Theorem 5.9].

Concerning the structure of $\Gamma_{g}$, the following is known.

Proposition 3.3 ([22]).

$$
\#\left(\Gamma_{g} /\left[\Gamma_{g}, \Gamma_{g}\right]\right)= \begin{cases}12 & (g=1), \\ 2 & (g=2), \\ 1 & (g>2) .\end{cases}
$$

Let $\Gamma^{\prime}$ be a cofinite subgroup of $\Gamma_{g}$ and $A\left(k, \chi, \Gamma^{\prime}\right)$ be the space of all modular forms of weight $k$ with character $\chi$ relative to the subgroup $\Gamma^{\prime}:$

$$
A\left(k, \chi, \Gamma^{\prime}\right)=\left\{f \in \mathcal{O}\left(\mathfrak{S}_{g}\right) ; f(\gamma \cdot \tau)=j(\tau, \gamma)^{k} \chi(\gamma) f(\tau), \quad \gamma \in \Gamma^{\prime}\right\}
$$


In particular, an element of $A_{k}(\Gamma):=A\left(k, 1, \Gamma_{g}\right)$ is called a Siegel modular form. The following modular form is important for us. Let $a, b \in B_{2}$. The parity of $\theta_{a, b}$ is defined by $4^{t} a \cdot b \in \mathbb{Z} / 2 \mathbb{Z}$. Set

$$
\chi_{g}(\tau):=\prod_{(a, b) \text { even }} \theta_{a, b}(0, \tau)
$$

It is known that $\chi_{1}(\tau)^{8}=2^{8} \Delta(\tau) \in A_{12}\left(\Gamma_{1}\right)$ ([12, pp.142]), $\chi_{2}(\tau)^{2} \in A_{10}\left(\Gamma_{2}\right)$, and $\chi_{g}(\tau) \in A_{2^{g-2}\left(2^{g}+1\right)}\left(\Gamma_{g}\right)$ for $g>2$ ([12, Chap.I, $3.3 \mathrm{Satz}])$. Finally, we remark that the function $\operatorname{det}(\operatorname{Im} \tau)$ has the following automorphic property:

$$
\operatorname{det} \operatorname{Im}(\gamma \cdot \tau)=|j(\tau, \gamma)|^{-2} \operatorname{det} \operatorname{Im} \tau
$$

\section{Ample divisors on Abelian varieties and determinant bundles}

Let $V_{m}=\mathbb{C}^{m^{g}}$ whose coordinates are denoted by $\left(u_{a}\right)_{a \in B_{m}}$. Let $\left\{\theta_{a}\right\}_{a \in B_{m}}$ be the basis of theta functions as in Proposition 3.1. Associated to $\left|L_{m}\right|$, let $\Theta_{m}$ be the family of ample divisors on Abelian varieties parametrised by $\mathbb{P}\left(V_{m}\right) \times \mathfrak{S}_{g}$ :

$$
\Theta_{m}:=\left\{(u, z, \tau) \in \mathbb{P}\left(V_{m}\right) \times \mathbb{A} ; \sum_{a \in B_{m}} u_{a} \theta_{a, 0}(m z, m \tau)=0\right\} .
$$

Set $\pi=i d_{\mathbb{P}\left(V_{m}\right)} \times p: \mathbb{P}\left(V_{m}\right) \times \mathbb{A} \rightarrow \mathbb{P}\left(V_{m}\right) \times \mathfrak{S}_{g}$. Its restriction to $\Theta_{m}$ is also denoted by $\pi$. The fiber $\Theta_{m,(u, \tau)}=\pi^{-1}(u, \tau)$ is a hypersurface on $A_{\tau}$ and all $\Theta_{m,(u, \tau)}$ are members of the same complete linear system $\left|L_{m, \tau}\right|$.

Since $\Theta_{1}=\Theta$ and $\mathbb{P}\left(V_{1}\right)$ is a point, we obtain the universal family of theta divisors when $m=1$. Furthermore, let $N_{g}$ be the Andreotti-Mayer locus, i.e., the discriminant of theta divisors:

$$
N_{g}:=\left\{\tau \in \mathfrak{S}_{g} ; \operatorname{Sing}\left(\Theta_{\tau}\right) \neq \emptyset\right\} .
$$

By Andreotti-Mayer, Beauville, Mumford, Smith-Varley, and finally Debarre, the following is known.

Proposition 4.1 ([9]). $\quad N_{g}$ is a divisor of $\mathfrak{S}_{g}$, consisting of two components:

$$
N_{g}=\theta_{n u l l, g}+2 N_{g}^{\prime},
$$


where $\theta_{\text {null, }, g}$ is the zero divisor of $\chi_{g}(\tau)$ (and $N_{g}^{\prime}=\emptyset$ when $g=2,3$ ). There exist proper subvarieties $Z_{1} \subset \theta_{\text {null,g }}$ and $Z_{2} \subset N_{g}^{\prime}$ such that

(1) For any $\tau \in \theta_{\text {null,g}}-Z_{1}$, Sing $\Theta_{\tau}$ consists of one $A_{1}$-singularity, i.e., a singularity whose local defining equation is $z_{1}^{2}+\cdots+z_{g}^{2}=0$.

(2) For any $\tau \in N_{g}^{\prime}-Z_{2}$, Sing $\Theta_{\tau}$ consists of two $A_{1}$-singularities which are mutually interchanged by the involution $x \rightarrow-x$.

In general, let

$$
\mathcal{D}_{g, m}:=\left\{(u, \tau) \in \mathbb{P}\left(V_{m}\right) \times \mathfrak{S}_{g} ; \text { Sing } \Theta_{m,(u, \tau)} \neq \emptyset\right\}
$$

be the discriminant locus of $\pi: \Theta_{m} \rightarrow \mathbb{P}\left(V_{m}\right) \times \mathfrak{S}_{g}$. Note that $\mathcal{D}_{g, 1}=$ $N_{g}$. Let $\mathcal{D}_{g, m, \tau}$ be the fiber at $\tau$ of the projection $p r_{2}: \mathcal{D}_{g, m} \rightarrow \mathfrak{S}_{g}$. Let $H_{m}=\mathcal{O}_{\mathbb{P}\left(V_{m}\right)}(1)$. Consider the morphism associated to the linear system $\left|p_{*} L_{m}\right|$ :

$$
\Phi_{m}:=\Phi_{\left|p_{*} L_{m}\right|}: \mathbb{A} \rightarrow \mathbb{P}\left(p_{*} L_{m}\right) \cong \mathbb{P}\left(V_{m}\right) \times \mathfrak{S}_{g} .
$$

By the Lefschetz theorem, we know the following. When $m=2, \Phi_{2}$ is a finite morphism. More precisely, $\Phi_{2}\left(A_{\tau}\right)$ is isomorphic to the Kummer variety $A_{\tau} /\{ \pm 1\}$ and $\Phi_{2}$ induces the projection map $A_{\tau} \rightarrow A_{\tau} / \pm 1$ on each fiber under this identification. When $m \geq 3, \Phi_{m}$ is an embedding. Since $L_{m}=\Phi_{m}^{*} H_{m}$, the support of $\mathcal{D}_{g, m, \tau}$ coincides with that of the discriminant locus of the linear system $\left|H_{m}\right|$ over $\Phi_{m}\left(A_{\tau}\right)$. As $H_{m}$ is the restriction of the hyperplane bundle, we get the following (when $m \geq 2$ ) by the general theory of Lefschetz pencil ([19, Théorème 2.5.2, Proposition 3.2, 3.3]).

Proposition 4.2. Suppose $m \geq 2$. Then, $\mathcal{D}_{g, m}$ is a divisor of $\mathbb{P}\left(V_{m}\right) \times \mathfrak{S}_{g}$. There exists a proper subvariety $Z_{g, m} \subset \mathcal{D}_{g, m}$ such that Sing $\Theta_{m,(u, \tau)}$ consists of $A_{1}$-singularities for any $(u, \tau) \in \mathcal{D}_{g, m}-Z_{g, m}$. Moreover, $\mathcal{D}_{g, m, \tau}$ is the projective dual variety of $\left(\Phi_{m}\left(A_{\tau}\right), H_{m}\right)$ for any $(u, \tau) \in \mathcal{D}_{g, m}-Z_{g, m}$.

Let $\lambda\left(\mathcal{O}_{\Theta_{m}}\right)=\otimes_{q \geq 0}\left(\operatorname{det} R^{q} \pi_{*} \mathcal{O}_{\Theta_{m}}\right)^{(-1)^{q}}$ be the determinant bundle. By Proposition $3.2, \Gamma_{g}(1,2)$ acts on $\mathbb{P}\left(V_{m}\right)$ via the representation $\rho_{m}: \Gamma_{g}(1,2) \rightarrow U\left(V_{m}\right)$ and thus on $\mathbb{P}\left(V_{m}\right) \times \mathbb{A}$. Furthermore it preserves $\Theta_{m}$, and therefore $\lambda\left(\mathcal{O}_{\Theta_{m}}\right)$ is endowed with a $\Gamma_{g}(1,2)$-module structure. Put $\omega:=p_{*} \omega_{\mathbb{A} / \mathfrak{S}_{g}}$.

Proposition 4.3. When $g>1$ and $m \geq 2$, there exists an isomorphism as $\mathcal{O}_{\mathbb{P}}\left(V_{m}\right) \times \mathfrak{S}_{g}$-modules with $\Gamma_{g}(1,2)$-action:

$$
\lambda\left(\mathcal{O}_{\Theta_{m}}\right)^{(-1)^{g}} \cong_{\Gamma_{g}(1,2)} \operatorname{det} \pi_{*} \omega_{\mathbb{A} / \mathfrak{G}_{g}}\left(\Theta_{m}\right) .
$$


Proof. Let $q: \mathbb{P}\left(p_{*} L_{m}\right) \rightarrow \mathfrak{S}_{g}$ be the projection to the second factor. Consider the following exact sequence of sheaves over $\mathbb{P}\left(V_{m}\right) \times \mathbb{A}$ :

$$
0 \longrightarrow \mathcal{O}_{\mathbb{P}\left(V_{m}\right) \times \mathbb{A}}\left(-\Theta_{m}\right) \longrightarrow \mathcal{O}_{\mathbb{P}\left(V_{m}\right) \times \mathbb{A}} \longrightarrow \mathcal{O}_{\Theta_{m}} \longrightarrow 0
$$

which, together with the relative Kodaira vanishing theorem, yields

$$
R^{i} \pi_{*} \mathcal{O}_{\Theta_{m}} \cong_{\Gamma_{g}(1,2)} R^{i} \pi_{*} \mathcal{O}_{\mathbb{P}\left(V_{m}\right) \times \mathbb{A}} \cong{ }_{\Gamma_{g}(1,2)} q^{*} R^{i} p_{*} \mathcal{O}_{\mathbb{A}} \quad(i<g-1),
$$

and

$$
\begin{aligned}
0 & \rightarrow R^{g-1} \pi_{*} \mathcal{O}_{\mathbb{P}\left(V_{m}\right) \times \mathbb{A}} \rightarrow R^{g-1} \pi_{*} \mathcal{O}_{\Theta_{m}} \\
& \rightarrow R^{g} \pi_{*} \mathcal{O}_{\mathbb{P}\left(V_{m}\right) \times \mathbb{A}}\left(-\Theta_{m}\right) \rightarrow R^{g} \pi_{*} \mathcal{O}_{\mathbb{P}\left(V_{m}\right) \times \mathbb{A}} \rightarrow 0 .
\end{aligned}
$$

Combining (4.5), (4.6) and the Serre duality

$$
R^{g} \pi_{*} \mathcal{O}_{\mathbb{P}\left(V_{m}\right) \times \mathbb{A}}\left(-\Theta_{m}\right) \cong_{\Gamma_{g}(1,2)}\left(\pi_{*} \omega_{\mathbb{A} / \mathfrak{S}_{g}}\left(\Theta_{m}\right)\right)^{\vee},
$$

we get

$$
\lambda\left(\mathcal{O}_{\Theta_{m}}\right) \cong_{\Gamma_{g}(1,2)} q^{*} \lambda\left(\mathcal{O}_{\mathbb{A}}\right) \otimes\left(\operatorname{det} \pi_{*} \omega_{\mathbb{A} / \mathfrak{S}_{g}}\left(\Theta_{m}\right)\right)^{(-1)^{g}} .
$$

Let $\lambda^{q}: \bigwedge^{q} R^{1} p_{*} \mathcal{O}_{\mathbb{A}} \rightarrow R^{q} p_{*} \mathcal{O}_{\mathbb{A}}$ be the homomorphism induced by the cup product of Dolbeaut cohomology groups. Comparing the dimension, we find that $\lambda^{q}$ is an isomorphism of $\mathcal{O}_{\mathfrak{S}_{g}}$-modules with $\Gamma_{g}$ action. Therefore,

$$
\lambda\left(\mathcal{O}_{\mathbb{A}}\right) \cong_{\Gamma_{g}} \bigotimes_{q \geq 0}\left(\operatorname{det} \bigwedge^{q} R^{1} p_{*} \mathcal{O}_{\mathbb{A}}\right)^{(-1)^{q}}
$$

Let $e=\left\{e_{1}, \cdots, e_{g}\right\}$ be a local frame of $R^{1} p_{*} \mathcal{O}_{\mathbb{A}}$. Fix an order in the set of index $\left\{J ; J=\left(j_{1}<\cdots<j_{q}\right)\right\}$. Under this order, put

$$
\sigma_{e}(\tau):=\bigotimes_{q \geq 0}\left(\bigwedge_{|J|=q} e_{J}\right)^{(-1)^{q}} \in \lambda\left(\mathcal{O}_{\mathbb{A}}\right)_{\tau}
$$

where $e_{J}:=e_{j_{1}} \wedge \cdots \wedge e_{j_{q}} \in \wedge^{q} R^{1} p_{*} \mathcal{O}_{\mathbb{A}}$ for $J=\left(j_{1}, \cdots, j_{q}\right)$. For $A \in G L(\mathbb{C}, g)$, put $A e:=\left\{A e_{1}, \cdots, A e_{g}\right\}$. Since $\lambda\left(\mathcal{O}_{\mathbb{A}}\right)$ is a line, there exists $f(A) \in \mathbb{C}^{*}$ such that $\sigma_{A e}=f(A) \sigma_{e}$. As is easily verified, $f: G L(\mathbb{C}, g) \rightarrow \mathbb{C}^{*}$ is a character and thus there exists $k \in \mathbb{Z}$ such that 
$f(A)=(\operatorname{det} A)^{k}$. Putting $A=x I$, we find $k=0$. (Here, we use $g>1$.) In particular, $\sigma_{e}$ does not depend on a choice of frames. Set

$$
1_{\mathbb{A}}(\tau):=\sigma_{e}(\tau)
$$

Then, $1_{\mathbb{A}}$ is a $\Gamma_{g}$-invariant section of $\lambda\left(\mathcal{O}_{\mathbb{A}}\right)$. In particular, $\lambda\left(\mathcal{O}_{\mathbb{A}}\right)$ is isomorphic to $\mathcal{O}_{\mathfrak{S}_{g}}$ as a $\Gamma_{g}$-module, and by (4.9),

$$
\lambda\left(\mathcal{O}_{\Theta_{m}}\right)^{(-1)^{g}} \cong_{\Gamma_{g}(1,2)} \operatorname{det} \pi_{*} \omega_{\mathbb{A} / \mathfrak{S}_{g}}\left(\Theta_{m}\right) .
$$

q.e.d.

To see the structure of $\operatorname{det} \pi_{*} \omega_{\mathbb{A} / \mathfrak{S}_{g}}\left(\Theta_{m}\right)$ as a $\Gamma_{g}(1,2)$-module, for any $c \in B_{m}$, we denote by $\mathcal{U}_{c}:=\left\{[u] \in \mathbb{P}\left(V_{m}\right) ; u_{c} \neq 0\right\}$ the open subset of $\mathbb{P}\left(V_{m}\right)$ which form a covering of $\mathbb{P}\left(V_{m}\right) ; \mathbb{P}\left(V_{m}\right)=\bigcup_{c \in B_{m}} \mathcal{U}_{c}$. Then, for any $(u, \tau) \in \mathcal{U}_{c} \times \mathfrak{S}_{g}$,

$$
\left\{\frac{u_{c} \theta_{a}}{\sum_{b \in B_{m}} u_{b} \theta_{b}} d z_{1} \wedge \cdots \wedge d z_{g}\right\}_{a \in B_{m}}
$$

is a $\mathbb{C}$-basis of $H^{0}\left(A_{\tau}, \Omega^{g}\left(\log \Theta_{m,(u, \tau)}\right)\right)$. Put

$$
s_{c}(u, \tau):=\bigwedge_{a \in B_{m}} \frac{u_{c} \theta_{a}}{\sum_{b \in B_{m}} u_{b} \theta_{b}} d z_{1} \wedge \cdots \wedge d z_{g}
$$

for a generator of $\operatorname{det} H^{0}\left(A_{\tau}, \Omega^{g}\left(\log \Theta_{m,(u, \tau)}\right)\right)$ when $(u, \tau) \in \mathcal{U}_{c} \times \mathfrak{S}_{g}$. Then, $s_{c}$ generates $\operatorname{det} \pi_{*} \omega_{\mathbb{A} / \mathfrak{S}_{g}}\left(\Theta_{m}\right)$ over $\mathcal{U}_{c} \times \mathfrak{S}_{g}$. For $u^{J}$ with $|J|=m^{g}$, define $\sigma_{J}$ on each $\mathcal{U}_{c} \times \mathfrak{S}_{g}$ by

$$
\sigma_{J} \mid{\mathcal{\mathcal { U } _ { c }} \times \mathfrak{S}_{g}}(u, \tau):=\frac{u^{J}}{u_{c}^{m^{g}}} s_{c}=u^{J} \cdot \bigwedge_{a \in B_{m}} \frac{\theta_{a}}{\sum_{b \in B_{m}} u_{b} \theta_{b}} d z_{1} \wedge \cdots \wedge d z_{g}
$$

Then, $\left.\sigma_{J}\right|_{\mathcal{U}_{c} \times \mathfrak{S}_{g}}=\left.\sigma_{J}\right|_{\mathcal{U}_{d} \times \mathfrak{S}_{g}}$ over $\mathcal{U}_{c} \cap \mathcal{U}_{d} \times \mathfrak{S}_{g}$ for any $c, d \in B_{m}$, and $\sigma_{J}$ becomes a global section, i.e.,

$$
\sigma_{J} \in H^{0}\left(\mathbb{P}\left(V_{m}\right) \times \mathfrak{S}_{g}, \operatorname{det} \pi_{*} \omega_{\mathbb{A} / \mathfrak{S}_{g}}\left(\Theta_{m}\right)\right) .
$$

Putting $J_{c}=\left(0, \cdots, m^{g}, \cdots, 0\right)$ (the $c$-th factor is $m^{g}$ and all the other factors vanish) in (4.16), we find that

$$
s_{c} \in H^{0}\left(\mathbb{P}\left(V_{m}\right) \times \mathfrak{S}_{g}, \operatorname{det} \pi_{*} \omega_{\mathbb{A} / \mathfrak{S}_{g}}\left(\Theta_{m}\right)\right) .
$$

As $s_{c}$ has no zero on $\mathcal{U}_{c} \times \mathfrak{S}_{g}$, we get the following. 
Proposition 4.4. When $g>1$ and $m \geq 2,\left\{\sigma_{J}\right\}_{|J|=m^{g}}$ generates $\operatorname{det} \pi_{*} \omega_{\mathbb{A} / \mathfrak{S}_{g}}\left(\Theta_{m}\right)$. Namely, the natural map

$$
\oplus_{a \in B_{m}} \mathcal{O}_{\mathbb{P}\left(V_{m}\right) \times \mathfrak{S}_{g}} \sigma_{J} \rightarrow \operatorname{det} \pi_{*} \omega_{\mathbb{A} / \mathfrak{S}_{g}}\left(\Theta_{m}\right)
$$

is surjective.

When $m=1$, we get the following.

Proposition 4.5. When $g>1$, there exists an isomorphism as $\mathcal{O}_{\mathfrak{S}_{g}}$-modules with $\Gamma_{g}(1,2)$-action:

$$
\lambda\left(\mathcal{O}_{\Theta}\right) \cong_{\Gamma_{g}(1,2)} \lambda\left(\mathcal{O}_{\mathbb{A}}\right) \otimes \omega^{(-1)^{g}} .
$$

In particular, $\lambda\left(\mathcal{O}_{\Theta}\right)$ has the following canonical section:

$$
\sigma_{\Theta}:=1_{\mathbb{A}} \otimes\left(d z_{1} \wedge \cdots \wedge d z_{g}\right)^{(-1)^{g}} .
$$

Proof. When $m=1$, the exact sequence (4.7) splits and the isomorphism (4.6) also holds for $i=g-1$ which implies the assertion. q.e.d.

\section{Ample divisors on Abelian varieties and Quillen metrics}

Let $p: \mathbb{A} \rightarrow \mathfrak{S}_{g}$ be the universal family of p.p.a.v., $p: \Theta \rightarrow \mathfrak{S}_{g}$ the universal family of theta divisors, and $\pi: \Theta_{m} \rightarrow \mathbb{P}\left(V_{m}\right) \times \mathfrak{S}_{g}$ the family of divisors associated to $\left|L_{m}\right|$ as before. Let

$$
\begin{gathered}
T \mathbb{A} / \mathfrak{S}_{g}:=\operatorname{ker} p_{*}, \\
T \Theta / \mathfrak{S}_{g}:=\left.\operatorname{ker} p_{*}\right|_{T \Theta}
\end{gathered}
$$

and

$$
T \Theta_{m} / \mathbb{P}\left(V_{m}\right) \times \mathfrak{S}_{g}:=\operatorname{ker} \pi_{*}
$$

be their relative tangent bundles. Clearly $T \Theta / \mathfrak{S}_{g}$ and $T \Theta_{m} / \mathbb{P}\left(V_{m}\right) \times \mathfrak{S}_{g}$ are subbundles of $T \mathbb{A} / \mathfrak{S}_{g}$. Let

$$
\begin{gathered}
\left.g_{\mathbb{A} / \mathfrak{S}_{g}}\right|_{A_{\tau}}={ }^{t} d z(\operatorname{Im} \tau)^{-1} d \bar{z}, \\
g_{\Theta / \mathfrak{S}_{g}}:=\left.g_{\mathbb{A} / \mathfrak{S}_{g}}\right|_{T \Theta / \mathfrak{S}_{g}},
\end{gathered}
$$


and

$$
g_{\Theta_{m} / \mathbb{P}\left(V_{m}\right) \times \mathfrak{S}_{g}}:=\left.g_{\mathbb{A} / \mathfrak{S}_{g}}\right|_{T \Theta_{m} / \mathbb{P}\left(V_{m}\right) \times \mathfrak{S}_{g}}
$$

be Hermitian metrics on $T \mathbb{A} / \mathfrak{S}_{g}, T \Theta / \mathfrak{S}_{g}$ and $T \Theta_{m} / \mathbb{P}\left(V_{m}\right) \times \mathfrak{S}_{g}$ which are invariant under the action of $\Gamma_{g}$ (resp. $\left.\Gamma_{g}(1,2)\right)$. Their restriction to each fiber is denoted by $g_{A_{\tau}}, g_{\Theta_{\tau}}$ and $g_{\Theta_{m,(u, \tau)}}$. Let $\|\cdot\|_{Q}$ be the Quillen metric of $\lambda\left(\mathcal{O}_{\Theta_{m}}\right)^{(-1)^{g}}$ relative to $g_{\Theta_{m} / \mathbb{P}\left(V_{m}\right) \times \mathfrak{S}_{q}}$ when $m>1$ and to $g_{\Theta / \mathfrak{S}_{g}}$ when $m=1$. By Propositions 4.4 and 4.5 , it is enough to know the Quillen norms for all $\sigma_{J}(m \geq 2)$ and $\sigma_{\Theta}(m=1)$ to understand $\|\cdot\|_{Q}$.

Theorem 5.1. Suppose $g>1$ and $m \geq 2$. There exist

$$
\Delta_{g, m}(u, \tau) \in \mathcal{O}\left(\mathfrak{S}_{g}\right)\left[u_{a}\right]_{a \in B_{m}}
$$

a homogeneous polynomial in u-variables of degree $m^{g} \cdot(g+1)$ ! with coefficients in $\mathcal{O}\left(\mathfrak{S}_{g}\right)$, and a character

$$
\chi_{g, m}: \Gamma_{g}(1,2) \rightarrow U(\mathbb{C})=S^{1}
$$

such that:

(1) For any $\gamma \in \Gamma_{g}(1,2)$ and $(u, \tau) \in \mathbb{P}\left(V_{m}\right) \times \mathfrak{S}_{g}$,

$$
\Delta_{g, m}(\gamma \cdot u, \gamma \cdot \tau)=\chi_{g, m}(\gamma) j(\tau, \gamma)^{\frac{1}{2}(g+3) \cdot g ! m^{g}} \Delta_{g, m}(u, \tau)
$$

(2) For any $J\left(|J|=m^{g}\right)$ and $(u, \tau) \in \mathbb{P}\left(V_{m}\right) \times \mathfrak{S}_{g}$,

$$
\left\|\sigma_{J}\right\|_{Q}^{2}(u, \tau)=(\operatorname{det} \operatorname{Im} \tau)^{\frac{(g-1) m^{g}}{2(g+1)}}\left|\frac{u^{J}}{\Delta_{g, m}(u, \tau)^{\frac{1}{(g+1) !}}}\right|^{2},
$$

(3) In the sense of divisor on $\mathbb{P}\left(V_{m}\right) \times \mathfrak{S}_{g}$, $\operatorname{div}\left(\Delta_{g, m}\right)=\mathcal{D}_{g, m}$.

Theorem 5.2. Let $\tau\left(\Theta_{\tau}\right)$ be the Ray-Singer analytic torsion of the smooth theta divisor $\left(\Theta_{\tau}, g_{\Theta_{\tau}}\right)$ of dimension $g-1(\geq 1)$. Then, there exists a Siegel cusp form $\Delta_{g}(\tau)$ of weight $\frac{(g+3) \cdot g !}{2}$ with zero divisor $N_{g}$ which vanishes at the highest dimensional cusp of order $\frac{(g+1) !}{12}$ such that

$$
\tau\left(\Theta_{\tau}\right)=\left\|\Delta_{g}(\tau)\right\|^{\frac{(-1)^{g+1} 2}{(g+1) !}} .
$$

For the proof of Theorems 5.1 and 5.2, we need several propositions. Assume $g>1$ in the sequel. 
Let $G \in \operatorname{Herm}_{+}(g)$ be a positive definite Hermitian matrix of type $(g, g)$, and $g_{G}:={ }^{t} d z G d \bar{z}$ a flat metric of $W:=\mathbb{C}^{g}$ associated to $G$. The identity matrix is denoted by $1_{g}$. Let $\mathbb{P}\left(W^{\vee}\right)$ be the projective space of hyperplanes of $W$, and $E$ be the universal vector bundle of rank $g-1$ over $\mathbb{P}\left(W^{\vee}\right)$. Namely, for $[a] \in \mathbb{P}\left(W^{\vee}\right), E_{[a]}$ is a hyperplane on $W$ corresponding to $[a]$. Consider the following exact sequence of vector bundles over $\mathbb{P}\left(W^{\vee}\right)$ :

$$
0 \longrightarrow E \longrightarrow W^{\vee}=\mathbb{C}^{g} \longrightarrow N=W^{\vee} / E \longrightarrow 0 .
$$

Note that $N=\mathcal{O}_{\mathbb{P}\left(W^{\vee}\right)}(1)$. Let $g_{E, G}:=\left.g_{G}\right|_{E}$ be the induced metric on E.

Proposition 5.1.

$$
\int_{\mathbb{P}\left(W^{\vee}\right)} \widetilde{\operatorname{Td}}\left(E ; g_{E, 1_{g}}, g_{E, G}\right)=\frac{(-1)^{g-1}(g-1)}{2(g+1) !} \log \operatorname{det} G .
$$

Proof. Put $H=\log G$ and $g_{t}:=g_{\exp (t H)}$ for the one-parameter family of metrics connecting $g_{1_{g}}$ and $g_{G}$. Its restriction to $E$ is denoted by $g_{E, t}$. Let $W^{\vee}=E \oplus_{t} E_{t}^{\perp}$ be the orthogonal decomposition of $W^{\vee}$ relative to $g_{t}$. Let $g_{N, t}$ be the metric of $N$ via the identification $N$ with $E_{t}^{\perp}$. Corresponding to this splitting, $H \in \operatorname{End}\left(W^{\vee}\right)$ can be written as follows:

$$
H=\left(\begin{array}{ll}
H_{11}(t) & H_{12}(t) \\
H_{21}(t) & H_{22}(t)
\end{array}\right)
$$

where $H_{11}(t) \in \operatorname{End}(E)$. Since $g_{E, t}\left(v_{1}, v_{2}\right)=g_{1_{g}}\left(\exp (t H) v_{1}, v_{2}\right)$ for any $v_{1}, v_{2} \in E$, we get

$$
g_{E, t}^{-1} \cdot \frac{d}{d t} g_{E, t}=H_{11} .
$$

Let $R_{E, t}$ be the curvature of $\left(E, g_{E, t}\right)$, and put $c_{1}\left(E_{t}\right):=\frac{i}{2 \pi} \operatorname{Tr} R_{E, t}$. By the Bott-Chern formula ([7, Proposition 3.15]), we find

$$
\widetilde{\operatorname{Td}}\left(E ; g_{E, 0}, g_{E, 1}\right)=\left.\int_{0}^{1} d t \frac{d}{d \epsilon}\right|_{\epsilon=0} \operatorname{Td}\left(\frac{i}{2 \pi} R_{E, t}+\epsilon g_{E, t}^{-1} \cdot \frac{d}{d t} g_{E, t}\right)
$$

Let $A_{t}$ be the second fundamental form of the exact sequence (5.1) relative to $g_{t}$. As $\left(W^{\vee}, g_{t}\right)$ is flat, by the Gauss-Codazzi equation ([21, Chap.I, (6.12)] and [28,(2.7)]), we obtain

$$
R_{E, t}=A_{t}^{*} \wedge A_{t}, \quad R_{N, t}=A_{t} \wedge A_{t}^{*}, \quad \operatorname{Tr} R_{E, t}^{k}=-R_{N, t}^{k},
$$


where $R_{N, t}$ is the curvature of $\left(N, g_{N, t}\right)$. Put $c_{1}\left(N_{t}\right):=\frac{i}{2 \pi} R_{N, t}$. Let $\operatorname{Td}_{k}(\cdot)$ be the homogeneous part of degree $k$ of the Todd polynomial. Then, there exists a polynomial $F\left(x_{1}, \cdots, x_{g-1}\right) \in \mathbb{Q}[x]$ such that, for any $X \in M(g-1, \mathbb{C})$,

$$
\operatorname{Td}_{g}(X)=F\left(\operatorname{Tr} X, \cdots, \operatorname{Tr} X^{g-1}\right) .
$$

By (5.3-6), we have

$$
\begin{aligned}
& {\left[\widehat{\operatorname{Td}}\left(E ; g_{E, 0}, g_{E, 1}\right)\right]^{(g-1, g-1)}} \\
& =\int_{0}^{1} d t\left[\sum_{j=1}^{g-1} j \frac{\partial F}{\partial x_{j}}\left(c_{1}\left(E_{t}\right), \cdots, c_{1}\left(E_{t}\right)^{g-1}\right) \operatorname{Tr}\left(H_{11}\left(\frac{i}{2 \pi} R_{E, t}\right)^{j-1}\right)\right]^{(g-1, g-1)} \\
& =\int_{0}^{1} d t\left[\sum_{j=1}^{g-1} j \frac{\partial F}{\partial x_{j}}\left(-c_{1}\left(N_{t}\right), \cdots,-c_{1}\left(N_{t}\right)^{g-1}\right)\left(\frac{i}{2 \pi}\right)^{j-1} \operatorname{Tr}\left(H_{11} R_{N, t}^{j-2} R_{E, t}\right)\right]^{(g-1, g-1)} \\
& =-\int_{0}^{1} d t\left[\sum_{j=1}^{g-1} j \frac{\partial F}{\partial x_{j}}\left(-c_{1}\left(N_{t}\right), \cdots,-c_{1}\left(N_{t}\right)^{g-1}\right) c_{1}\left(N_{t}\right)^{j-2} \wedge \frac{i}{2 \pi} A_{t} H_{11} A_{t}^{*}\right]^{(g-1, g-1)}
\end{aligned}
$$

where

$$
\operatorname{Tr}\left(H_{11} R_{N, t}^{-1} R_{E, t}\right)=-R_{N, t}^{-1} \cdot A_{t} H_{11} A_{t}^{*}=\operatorname{Tr} H_{11}
$$

for $j=1$ in the second and the third equality of (5.7). Since $H_{11}(t)$ is a Hermitian matrix, we can write, by an appropriate choice of a frame at $p$,

$$
H_{11}(t, p)=\left(\begin{array}{ccc}
\rho_{1} & & \\
& \ddots & \\
& & \rho_{g-1}
\end{array}\right)
$$

with some $\rho_{1}, \cdots, \rho_{g-1} \in \mathbb{R}$. Let $A_{t}=\left(a_{1}, \cdots, a_{g-1}\right)$ be the second fundamental form. Let $c(g)$ be the constant which depends only on $g$ such that

$$
\left.\sum_{j=2}^{g-1} j \frac{\partial F}{\partial x_{j}}\left(-x, \cdots,-x^{g-1}\right) x^{j-2}\right|_{x^{g-2}}=c(g)
$$

where $\left.h(x)\right|_{x^{g}}$ is the coefficient of $x^{g}$ for $h(x) \in \mathbb{C}[[x]]$. Since $R_{N, t}=$ 
$\sum a_{i} \wedge \bar{a}_{i}$ by $(5.5)$, we get

$$
\begin{aligned}
& {\left[\sum_{j=2}^{g-1} j \frac{\partial F}{\partial x_{j}}\left(-c_{1}\left(N_{t}\right), \cdots,-c_{g-1}\left(N_{t}\right)\right) c_{1}\left(N_{t}\right)^{j-2} \wedge A_{t} H_{11} A_{t}^{*}\right]^{(g-1, g-1)}} \\
& \quad=c(g)\left(\frac{i}{2 \pi} \sum_{i=1}^{g-1} a_{i} \wedge \bar{a}_{i}\right)^{g-2} \wedge \sum_{i=1}^{g-1} \frac{i}{2 \pi} \rho_{i} a_{i} \wedge \bar{a}_{i} \\
& \quad=\frac{\sum_{i} \rho_{i}}{g-1} c(g)\left(\frac{i}{2 \pi} \sum_{i} a_{i} \wedge \bar{a}_{i}\right)^{g-1} \\
& \quad=\frac{\operatorname{Tr} H_{11}}{g-1}\left[\sum_{j=2}^{g-1} j \frac{\partial F}{\partial x_{j}}\left(-c_{1}\left(N_{t}\right), \cdots,-c_{g-1}\left(N_{t}\right)\right) c_{1}\left(N_{t}\right)^{j-1}\right]^{(g-1, g-1)}
\end{aligned}
$$

Separating the summation of the third equality of (5.7) into that for $j=1$ and for $j \geq 2$, and substituting (5.8) and (5.11) respectively, we get

$$
\begin{aligned}
\left.\widetilde{\mathrm{Td}}\left(E ; g_{E, 0}, g_{E, 1}\right)\right]^{(g-1, g-1)} \\
=\int_{0}^{1} \operatorname{Tr} H_{11}(t) \frac{\partial F}{\partial x_{1}}\left(c_{1}\left(E_{t}\right), \cdots, c_{1}\left(E_{t}\right)^{g-1}\right) d t \\
\quad+\frac{1}{g-1} \int_{0}^{1} \operatorname{Tr} H_{11}(t) \sum_{j=2}^{g-1} j \frac{\partial F}{\partial x_{j}} \\
\left.=\left.\frac{1}{g-1} \int_{0}^{1} \operatorname{Tr} H_{11}(t) \frac{d}{d \epsilon}\right|_{\epsilon=0}\left(E_{t}\right), \cdots, c_{1}\left(E_{t}\right)^{g-1}\right) c_{1}\left(E_{t}\right)^{j-1} d t \\
=\frac{1}{g-1} \int_{0}^{1} \operatorname{Tr} H_{11}(t) \operatorname{Td}^{\prime}\left(R_{E, t}\right)^{(g-1, g-1)} d t \\
=\frac{1}{g-1} \operatorname{Tr} H \int_{0}^{1} \operatorname{Td}^{\prime}\left(R_{E, t}\right)^{(g-1, g-1)} d t \\
\quad-\frac{1}{g-1} \int_{0}^{1} H_{22}(t) \operatorname{Td}^{\prime}\left(R_{E, t}\right)^{(g-1, g-1)} d t
\end{aligned}
$$

where $\operatorname{Td}^{\prime}\left(R_{E, t}\right):=\left.\frac{d}{d \epsilon}\right|_{\epsilon=0} \operatorname{Td}\left(\epsilon 1_{g-1}+\frac{i}{2 \pi} R_{E, t}\right)$. 
Put $f(x):=x^{-1}-e^{-x}\left(1-e^{-x}\right)^{-1}$. As $\operatorname{Td}^{-1}(x)=\left(1-e^{-x}\right) x^{-1}$, we get

$$
\left.\mathrm{Td}^{-1}(x)\{g \cdot f(0)-f(x)\}\right|_{x^{g-1}}=\frac{(-1)^{g+1} g(g-1)}{2(g+1) !} .
$$

Using (5.5), we can show that $\operatorname{Td}\left(\frac{i}{2 \pi} R_{E, t}\right) \operatorname{Td}\left(c_{1}\left(N_{t}\right)\right)=1$ (cf. $[28$, (2.8)]) which, together with [5, Proposition 4.4] and (5.13), yields

$$
\begin{aligned}
\operatorname{Td}^{\prime}\left(R_{E, t}\right) & =\operatorname{Td}\left(\frac{i}{2 \pi} R_{E, t}\right) \operatorname{Tr} f\left(\frac{i}{2 \pi} R_{E, t}\right) \\
& =\operatorname{Td}^{-1}\left(c_{1}\left(N_{t}\right)\right)\left\{g \cdot f(0)-f\left(c_{1}\left(N_{t}\right)\right)\right\} \\
& =\frac{(-1)^{g+1} g(g-1)}{2(g+1) !} c_{1}\left(N_{t}\right)^{g-1} .
\end{aligned}
$$

Comparing (5.12) and (5.14) leads to

$$
\begin{aligned}
\int_{\mathbb{P}\left(W^{\vee}\right)} & \widetilde{\operatorname{Td}}\left(E ; g_{E, 0}, g_{E, 1}\right) \\
= & \frac{(-1)^{g+1} g}{2(g+1) !}\left(\operatorname{Tr} H-\int_{0}^{1} d t \int_{\mathbb{P}\left(W^{\vee}\right)} H_{22}(t) c_{1}\left(N_{t}\right)^{g-1}\right) .
\end{aligned}
$$

Let us compute $H_{22}(t)$. In the sequel, identify $W=W^{\vee}=\mathbb{C}^{g}$. For $z \in \mathbb{C}^{g}$,

$$
E_{z}=\left\{u \in \mathbb{C}^{g} ; \sum_{i=1}^{g} u_{i} z_{i}=0\right\}
$$

Since $g_{t}(u, v)={ }^{t} u \exp (t H) \bar{v}$, we find $E_{z}^{\perp}=\mathbb{C} \exp (-t H) \bar{z}$. By a suitable choice of coordinates, we may assume

$$
\begin{aligned}
G z & =\left(\lambda_{1} z_{1}, \cdots, \lambda_{g} z_{g}\right), \\
H z & =\left(\mu_{1} z_{1}, \cdots, \mu_{g} z_{g}\right), \\
\lambda_{i} & =\exp \left(\mu_{i}\right) .
\end{aligned}
$$

In above coordinates,

$$
H_{22}(t)=g_{N, t}^{-1} \cdot \frac{d}{d t} g_{N, t}=\frac{\sum_{i=1}^{g} \mu_{i} e^{-t \mu_{i}}\left|z_{i}\right|^{2}}{\sum_{i=1}^{g} e^{-t \mu_{i}}\left|z_{i}\right|^{2}}
$$


Put $w_{i}:=\exp \left(-\frac{1}{2} \mu_{i} t\right) z_{i}$ and $\omega_{\mathbb{P}^{g-1}}:=\frac{i}{2 \pi} \partial \bar{\partial} \log \sum\left|w_{i}\right|^{2}$. From (5.15) and (5.18), it follows that

$$
\begin{aligned}
\int_{\mathbb{P}\left(V^{\vee}\right)} & \widetilde{\operatorname{Td}}\left(E ; g_{E, 0}, g_{E, 1}\right) \\
= & \frac{(-1)^{g+1} g}{2(g+1) !}\left(\operatorname{Tr} H-\int_{0}^{1} d t \int_{\mathbb{P}^{g-1}} \frac{\sum_{i=1}^{g} \mu_{i}\left|w_{i}\right|^{2}}{\sum_{i=1}^{g}\left|w_{i}\right|^{2}} \omega_{\mathbb{P}^{g-1}}^{g-1}\right) \\
= & \frac{(-1)^{g+1}(g-1)}{2(g+1) !} \operatorname{Tr} H,
\end{aligned}
$$

which, combined with $\operatorname{Tr} H=\log \operatorname{det} G$, yields the assertion. q.e.d.

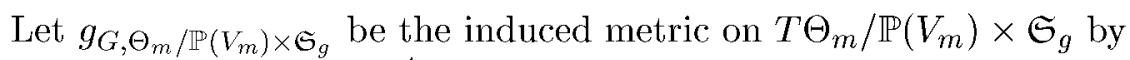
the constant metric $g_{G}={ }^{t} d z G d \bar{z}$ on $T \mathbb{A} / \mathfrak{S}_{g}$ where $G \in \operatorname{Herm}_{+}(g)$. Let $\|\cdot\|_{Q, G}$ be the Quillen metric of $\lambda\left(\mathcal{O}_{\Theta_{m}}\right)$ relative to $g_{G, \Theta_{m} / \mathbb{P}\left(V_{m}\right) \times \mathfrak{S}_{g}}$; its restriction to each fiber is denoted by $g_{G, \Theta_{m,(u, \tau)}}$. Remember that $\|\cdot\|_{Q}$ is the Quillen metric of $\lambda\left(\mathcal{O}_{\Theta_{m}}\right)$ relative to the invariant metric $g_{\tau}={ }^{t} d z(\operatorname{Im} \tau)^{-1} d \bar{z}$ of $A_{\tau}$ (see the beginning of this section).

\section{Proposition 5.2.}

$$
\log \frac{\|\cdot\|_{Q}^{2}}{\|\cdot\|_{Q, 1_{g}}^{2}}(\tau)=\frac{(-1)^{g}(g-1) m^{g}}{2(g+1)} \log \operatorname{det} \operatorname{Im} \tau .
$$

Proof. Let $\nu: \Theta_{m,(u, \tau)} \rightarrow \mathbb{P}\left(V_{m}\right)$ be the Gauss map:

$$
\nu: \Theta_{m,(u, \tau)} \ni z \longrightarrow\left(T \Theta_{m,(u, \tau)}\right)_{z} \in \mathbb{P}\left(V_{m}\right),
$$

which is a finite covering with mapping degree $m^{g} g !$. By definition,

$$
\left(T \Theta_{m,(u, \tau)}, g_{G, \Theta_{m,(u, \tau)}}\right)=\nu^{*}\left(E, g_{E, G}\right),
$$

which, together with Theorem 2.2 and Proposition 5.2, implies

$$
\begin{aligned}
\log \frac{\|\cdot\|_{Q, G}^{2}}{\|\cdot\|_{Q, 1_{g}}^{2}}(u, \tau) & =\int_{\Theta_{m,(u, \tau)}} \nu^{*} \widetilde{\mathrm{Td}}\left(E ; g_{E, 1_{g}}, g_{E, G}\right) \\
& =\operatorname{deg} \nu \int_{\mathbb{P}\left(V_{m}\right)} \widetilde{\mathrm{Td}}\left(E ; g_{E, 1_{g}}, g_{E, G}\right) \\
& =\frac{(-1)^{g+1}(g-1) m^{g}}{2(g+1)} \log \operatorname{det} G
\end{aligned}
$$

The assertion follows from (5.22) by putting $G=(\operatorname{Im} \tau)^{-1}$. q.e.d. 
Let

$$
\Sigma_{m}:=\left\{x \in \Theta_{m} ; x \in \operatorname{Sing} \Theta_{(u, \tau)}, \quad \pi(x)=(u, \tau)\right\}
$$

be the singular locus of

$$
\pi: \Theta_{m} \rightarrow \mathbb{P}\left(V_{m}\right) \times \mathfrak{S}_{g}
$$

Thus, $\mathcal{D}_{g, m}=\pi\left(\Sigma_{m}\right)$.

Proposition 5.3 ([28, Proposition 2.1]). Outside of $\Sigma_{m}$, the following holds:

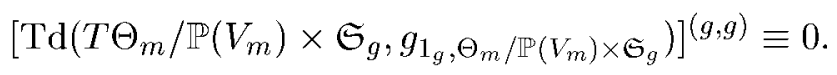

In particular, one has

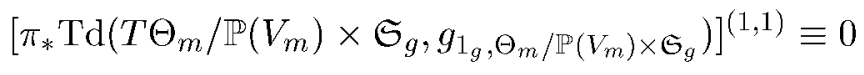

over $\mathbb{P}\left(V_{m}\right) \times \mathfrak{S}_{g} \backslash \mathcal{D}_{g, m}$ and its trivial extension to $\mathbb{P}\left(V_{m}\right) \times \mathfrak{S}_{g}$ is smooth.

Proof of Theorem 5.1. Let

$$
\sigma_{J} \in H^{0}\left(\mathbb{P}\left(V_{m}\right) \times \mathfrak{S}_{g}, \lambda\left(\mathcal{O}_{\Theta_{m}}\right)^{(-1)^{g}}\right)
$$

be the same as in (4.16). As is easily verified,

$$
F_{m}(u, \tau):=\frac{\left\|\sigma_{J}\right\|_{Q, 1_{g}}^{2}}{\left|u^{J}\right|^{2}}=(\operatorname{det} \operatorname{Im} \tau)^{-\frac{(g-1) m^{g}}{2(g+1)}} \frac{\left\|\sigma_{J}\right\|_{Q}^{2}}{\left|u^{J}\right|^{2}}
$$

is a function on $V_{m} \times \mathfrak{S}_{g}$ independent of a choice of index $J$. (Note that $(-1)^{g}$ does not enter into $(5.23)$ because we consider $\lambda\left(\mathcal{O}_{\Theta_{m}}\right)^{(-1)^{g}}$ rather than $\lambda\left(\mathcal{O}_{\Theta_{m}}\right)$.) For any $\gamma \in \Gamma_{g}(1,2)$, we get

$$
\gamma \cdot \sigma_{J}=\operatorname{det} \rho_{m} \cdot\left(\frac{\tilde{\rho}_{m}(\gamma) \cdot u^{J}}{u^{J}}\right) \cdot \sigma_{J}
$$

where

$$
\tilde{\rho}_{m}: \Gamma_{g}(1,2) \rightarrow \operatorname{End}\left(\operatorname{Sym}^{r}\left(V_{m}\right)\right)
$$

and

$$
\operatorname{det} \rho_{m}: \Gamma_{g}(1,2) \rightarrow U\left(\operatorname{det} V_{m}\right)=U(\mathbb{C})
$$


are the induced representation from that of Proposition 3.2. Since $\|\cdot\|_{Q}$ is invariant under the action of $\Gamma_{g}(1,2)$, it follows from $(3.7),(5.23)$ and (5.24) that

$$
\begin{aligned}
F_{m}(\gamma \cdot u, \gamma \cdot \tau) & =(\operatorname{det} \operatorname{Im}(\gamma \cdot \tau))^{-\frac{(g-1) m^{g}}{2(g+1)}} \frac{\left\|\gamma \cdot \sigma_{J}\right\|_{Q}^{2}}{\left|\tilde{\rho}_{m}(\gamma) \cdot u^{J}\right|^{2}} \\
& =|j(\tau, \gamma)|^{-\frac{g+3}{g+1} m^{g}} F_{m}(u, \tau)
\end{aligned}
$$

Let

$$
c: S=\{t \in \mathbb{C} ;|t|<1\} \ni t \rightarrow(u(t), \tau(t)) \in \mathbb{P}\left(V_{m}\right) \times \mathfrak{S}_{g}
$$

be an arbitrary holomorphic curve which intersects $\mathcal{D}_{g, m}$ transversally at $t=0$, and $(u(0), \tau(0))$ is a generic point of $\mathcal{D}_{g, m}$ in the sense of Proposition 4.2, i.e., $(u(0), \tau(0)) \in \mathcal{D}_{g, m}-Z_{g, m}$. Applying Theorems 2.1, 2.3 and Proposition 5.3 to the family $S \times \mathbb{P}\left(V_{m}\right) \times \mathcal{S}_{g} \Theta_{m}$, we get

$$
\begin{array}{r}
F_{m}(u(t), \tau(t))=\operatorname{mult}_{(u(0), \tau(0))} \mathcal{D}_{g, m} \cdot \log |t|^{2}+\psi(t), \\
\psi(t) \in C^{\infty}(S)
\end{array}
$$

which, combined with Proposition 5.3 and the argument in [2, Proposition 10.2], yields the following equation of currents over $V_{m} \times \mathfrak{S}_{g}$ :

$$
\frac{i}{2 \pi} \bar{\partial} \partial \log F_{m}(u, \tau)=\frac{1}{(g+1) !} \Pi^{*} \delta_{\mathcal{D}_{g, m}}=\frac{1}{(g+1) !} \delta_{\Pi^{*} \mathcal{D}_{g}, m},
$$

where $\Pi:\left(V_{m}-\{0\}\right) \times \mathfrak{S}_{g} \rightarrow \mathbb{P}\left(V_{m}\right) \times \mathfrak{S}_{g}$ is the natural projection, and $\delta_{\mathcal{D}_{g, m}}$ is the current corresponding to the integration along $\mathcal{D}_{g, m}$. Since $V_{m} \times \mathfrak{S}_{g}$ is a Stein manifold diffeomorphic to the Euclidean space, there exists a holomorphic function $\Delta_{g, m}(u, \tau) \in \mathcal{O}\left(V_{m} \times \mathfrak{S}_{g}\right)$ such that

$$
\left|\Delta_{g, m}(u, \tau)\right|^{2}=F_{m}(u, \tau)^{-(g+1) !} .
$$

As $\mathcal{D}_{g, m, \tau}$ is a projective hypersurface, $\Delta_{g, m}(\cdot, \tau)$ must be its defining homogeneous polynomial because $F_{m}(u, \tau)$ is a homogeneous function in $u$-variable. Put

$$
\chi_{g, m}(\gamma, u, \tau):=\frac{\Delta_{g, m}(\gamma \cdot u, \gamma \cdot \tau)}{j(\tau, \gamma)^{\frac{(g+3) \cdot g ! \cdot m g}{2}} \Delta_{g, m}(u, \tau)} .
$$

By (5.25) and (5.28), $\left|\chi_{g, m}(\gamma, u, \tau)\right|=1$ for any $(u, \tau) \in V_{m} \times \mathfrak{S}_{g}$ and thus $\chi_{g, m}(\gamma, u, \tau)=\chi_{g, m}(\gamma)$ for some $\chi_{g, m}(\gamma) \in U(\mathbb{C})$. Since $j(\tau, \gamma)$ is an automorphic factor, $\chi_{g, m}: \Gamma_{g}(1,2) \rightarrow U(\mathbb{C})$ is a character, which 
together with (5.29) implies Theorem 5.1 (1). Theorem 5.1 (3) follows from (5.27) and (5.28). Since

$$
\left\|\sigma_{J}\right\|_{Q}^{2}=(\operatorname{det} \operatorname{Im} \tau)^{\frac{(g-1) m^{g}}{2(g+1)}}\left|\frac{u^{J}}{\Delta_{g, m}(u, \tau)^{\frac{1}{(g+1) !}}}\right|^{2}
$$

by (5.23) and (5.28), we obtain Theorem 5.1 (2). q.e.d.

Proof of Theorem 5.2. In the same way as the proof of Theorem 5.1 , there exists a modular form $\Delta_{g}(\tau) \in A\left(\frac{(g+3) \cdot g !}{2}, \chi, \Gamma_{g}(1,2)\right)$ such that

$$
\left\|\sigma_{\Theta}\right\|_{Q}^{2}(\tau)=(\operatorname{det} \operatorname{Im} \tau)^{\frac{(-1)^{g}(g-1)}{2(g+1)}}\left|\Delta_{g}(\tau)\right|^{\frac{2(-1)^{g+1}}{(g+1) !}} .
$$

At first, let us verify that $\Delta_{g}(\tau)$ is a modular form with respect to the full Siegel modular group $\Gamma_{g}$. For $\gamma \in \Gamma_{g}$, put

$$
\phi_{\gamma}(\tau):=\left|\frac{\Delta_{g}(\gamma \cdot \tau)}{j(\tau, \gamma)^{\frac{(g+3) \cdot g !}{2}} \Delta_{g}(\tau)}\right|^{2} .
$$

As is easily verified, $\phi_{\gamma}(\tau)$ depends only on $[\gamma] \in \Gamma_{g} / \Gamma_{g}(1,2)$. Furthermore, for any $g \in \Gamma_{g}(1,2), \phi_{\gamma}(g \cdot \tau)=\phi_{\gamma g}(\tau)$. Since $N_{g}$ is invariant under the action of $\Gamma_{g}, \phi_{\gamma}$ is a plurisubharmonic function over $\mathfrak{S}_{g}$ without any zero and pole. Therefore, if $A\left(x_{[\gamma]}\right)$ is an elementary symmetric polynomial of $\left\{x_{[\gamma]}\right\}_{[\gamma] \in \Gamma_{g}(1,2) \backslash \Gamma_{g}}, A\left(\phi_{[\gamma]}(\tau)\right)$ is a $\Gamma_{g}(1,2)$-invariant plurisubharmonic function on $\mathfrak{S}_{g}$ and thus descends to a plurisubharmonic function on $\mathfrak{S}_{g} / \Gamma_{g}(1,2)$. As $g>1, A\left(\phi_{[\gamma]}(\tau)\right)$ extends to the Satake compactification ([13]) and should be a constant. In particular, any $\phi_{\gamma}(\tau)$ is a constant. Put

$$
\tilde{\chi}(\gamma):=\frac{\Delta_{g}(\gamma \cdot \tau)}{j(\tau, \gamma)^{\frac{(g+3) \cdot g^{\prime}}{2}} \Delta_{g}(\tau)} .
$$

As before, $\tilde{\chi}: \Gamma_{g} \rightarrow \mathbb{C}^{\times}$is a character which coincides with $\chi$ restricted to $\Gamma_{g}(1,2)$. It is a $U(\mathbb{C})$-character, because $\Gamma_{g} / \Gamma_{g}(1,2)$ is finite. From Proposition 3.3 , it follows that $\tilde{\chi}=1$ when $g>2$ and $\tilde{\chi}= \pm 1$ when $g=2$ which shows that $\Delta_{g}(\tau)$ is a Siegel modular form relative to $\Gamma_{g}$ (with character when $g=2$ ). By Mumford's formula ([24, Theorem $2.10])$, it is immediate that $\Delta_{g}(\tau)$ vanishes at the highest dimensional cusp of order $\frac{(g+1) !}{12}$. 
Let us compute the $L^{2}$-norm of $\sigma_{\Theta}$. Let $\mathcal{H}^{0,1}\left(A_{\tau}\right)$ be the space of harmonic $(0,1)$-forms on $A_{\tau}$. Identify $\mathcal{H}^{0,1}\left(A_{\tau}\right) \cong H^{1}\left(A_{\tau}, \mathcal{O}_{A_{\tau}}\right)$ and let $\omega_{1}, \cdots, \omega_{g}$ be a basis of $\mathcal{H}^{0,1}\left(A_{\tau}\right)$ such that $\int_{A_{\tau}} d z_{1} \wedge \cdots \wedge d z_{g} \wedge \omega_{1} \wedge$ $\cdots \wedge \omega_{g}=1$, i.e.,

$$
\omega_{1} \wedge \cdots \wedge \omega_{g}=\left(\frac{i}{2}\right)^{g} \frac{d \bar{z}_{1} \wedge \cdots \wedge d \bar{z}_{g}}{\operatorname{det} \operatorname{Im} \tau}
$$

For $I=\left(i_{1}, \cdots, i_{p}\right)$ put $\omega_{I}:=\omega_{i_{1}} \wedge \cdots \wedge \omega_{i_{p}}$ and $\omega^{(q)}:=\bigwedge_{|I|=q} \omega_{I} \in$

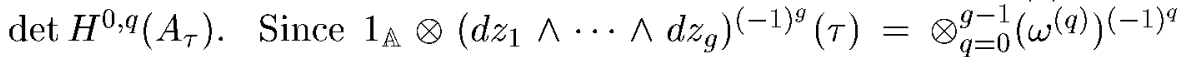
and $c_{1}\left(L_{\tau}\right)$ is cohomologous to $\delta_{\Theta_{\tau}}$, we get the assertion by combining Definition 2.1, (5.31) and the following:

$$
\begin{aligned}
\log & \left\|\sigma_{\Theta}\right\|_{L^{2}\left(\Theta_{\tau}\right)}^{2}(\tau) \\
& =\sum_{q=0}^{g-1}(-1)^{q} \log \left|\operatorname{det}\left(\int_{\Theta_{\tau}} \omega_{I} \wedge \bar{\omega}_{J} \wedge c_{1}\left(L_{\tau}\right)^{g-q-1}\right)_{|I|=|J|=q}\right| \\
& =\sum_{q=0}^{g-1}(-1)^{q} \log \left|\operatorname{det}\left(\int_{A_{\tau}} \omega_{I} \wedge \bar{\omega}_{J} \wedge c_{1}\left(L_{\tau}\right)^{g-q}\right)_{|I|=|J|=q}\right| \\
& =(-1)^{g} \log (\operatorname{det} 2 \operatorname{Im} \tau) . \quad \text { q.e.d. }
\end{aligned}
$$

Remark. It is worth noting that Theorem 2.4 yields the following integral representation fromula for $\Delta_{g}(\tau)$ :

$$
\begin{aligned}
\log \left|\Delta_{g}(\tau)\right|^{2} & \\
= & \int_{\Theta_{\tau}} \sum_{i+j=g-1} c_{1}\left(L_{\tau}\right)^{i} \wedge \nu_{\tau}^{*} c_{1}\left(H_{\tau}\right)^{j} \log \|d \theta\|_{\Omega_{A_{\tau}}^{1} \mid \Theta_{\tau} \otimes L_{\tau}}^{2} \\
& +\int_{A_{\tau}} \log \|\theta\|_{L_{\tau}}^{2} c_{1}\left(L_{\tau}\right)^{g}-g ! \log \operatorname{det} \operatorname{Im} \tau+C(g),
\end{aligned}
$$

where $c_{1}\left(H_{\tau}\right)=\frac{i}{2 \pi} \partial \bar{\partial} \log ^{t} z(\operatorname{Im} \tau) \bar{z}$ is the Fubini-Study form of $\mathbb{P}^{g-1}$, $C(g)$ a constant depending only on $g$ and $\nu_{\tau}: \Theta_{\tau} \rightarrow \mathbb{P}^{g-1}$ is the Gauss map. Note that the formula (5.36) for $g=1$ implies Faltings's formula $([10])$ :

$$
\int_{E_{\tau}} \log \|\theta(z, \tau)\|_{L_{\tau}}^{2} c_{1}\left(L_{\tau}\right)=\log |\Delta(\tau)|^{\frac{1}{12}}
$$

where $\Delta(\tau)$ is the Jacobi's $\Delta$-function. 


\section{Projective duality and structure of $\Delta_{g, m}(u, \tau)$}

Throughout this section, let us assume $g>1$. By Theorem 5.1, there exists a holomorphic function $f_{J}(\tau) \in \mathcal{O}\left(\mathfrak{S}_{g}\right)$ for any $J(|J|=$ $\left.m^{g} \cdot(g+1) !\right)$ such that

$$
\Delta_{g, m}(u, \tau)=\sum_{J} f_{J}(\tau) u^{J}
$$

Among all the elements of $B_{m}$, there exists a special one 0 . We write $u=\left(u_{0}, u^{\prime}\right)$ where $u^{\prime}=\left(u_{a}\right), a \in B_{m} \backslash\{0\}$. Under this notation, $J_{0}:=$ $\left(m^{g} \cdot(g+1) !, 0, \cdots, 0\right)$ satisfies $u^{J_{0}}=u_{0}^{m^{g} \cdot(g+1) !}$. Since both $\Delta_{g}(\tau)$ and $\Delta_{g, m}(u, \tau)$ have an ambiguity of complex numbers of modulus one, we impose them the condition that $\Delta_{g}\left(\tau_{0}\right)>0$ and $f_{J_{0}}\left(\tau_{0}\right)>0$ at some $\tau_{0} \in \mathfrak{S}_{g}$.

Theorem 6.1. For any $\tau \in \mathfrak{S}_{g}$,

$$
f_{J_{0}}(\tau)=f_{\left(m^{g} \cdot(g+1) !, 0, \cdots, 0\right)}(\tau)=m^{\frac{g \cdot g ! m^{g}}{2}} \Delta_{g}(m \tau)^{m^{g}} .
$$

Proof. To relate $A_{\tau}$ and $A_{m \tau}$, let $\mu_{m}$ be the isogeny of Abelian varieties defined by $\mu_{m}: A_{\tau} \ni[z] \rightarrow[m z] \in A_{m \tau}$ whose kernel is isomorphic to $(\mathbb{Z} / m \mathbb{Z})^{g}$. Thus, $\mu_{m}: A_{\tau} \rightarrow A_{m \tau}$ is an unramified covering of mapping degree $m^{g}$. Let $\Theta_{m,((1,0), \tau)}$ be the divisor on $A_{\tau}$ defined by $\Theta_{m,((1,0), \tau)}=\left\{z \in A_{\tau} ; \theta(m z, m \tau)=0\right\}$. By definition, it is clear that $\Theta_{m,((1,0), \tau)}=\mu_{m}^{-1} \Theta_{m \tau}$ and $\mu_{m}: \Theta_{m,((1,0), \tau)} \rightarrow \Theta_{m \tau}$ is an unramified covering of degree $m^{g}$ where $\Theta_{m \tau}$ is the theta divisor of $A_{m \tau}$. By Proposition 3.1, $\theta_{\frac{0}{m}}(\tau):=\theta(m z, m \tau)$ is a global section of $L_{\tau}^{m}:=L_{\tau}^{\otimes m}$ which is equipped with the Hermitian metric defined by (3.5). It is easy to verify the following:

$$
\begin{aligned}
\mu_{m}^{*} \theta(\cdot, m \tau) & =\theta_{\frac{0}{m}}(\tau), \\
\mu_{m}^{*}\left(L_{m \tau}, h_{L_{m \tau}}\right) & =\left(L_{\tau}^{\otimes m}, h_{L_{\tau}^{m}}\right), \\
\mu_{m}^{*} g_{m \tau} & =m g_{\tau},
\end{aligned}
$$

where $g_{\tau}={ }^{t} d z(\operatorname{Im} \tau)^{-1} d \bar{z}$ is the Kähler metric of $A_{\tau}$. Put $N^{\prime}:=$ $N_{\Theta_{m,((1,0), \tau)} / A_{\tau}}$ and $N:=N_{\Theta_{m \tau} / A_{m \tau}}$ which are equipped with the Hermitian metrics $g_{N^{\prime}}$ and $g_{N}$ such that

$$
\left\|d \theta_{\frac{0}{m}}(\tau)\right\|_{N^{\prime}-1 \otimes L_{\tau}^{-m}}^{2} \equiv 1, \quad\|d \theta(\cdot, m \tau)\|_{N^{-1} \otimes L_{m \tau}^{-1}}^{2} \equiv 1
$$


on $\Theta_{m,\{(1,0), \tau)}$ and $\Theta_{m \tau}$ respectively. Let

$$
\overline{\mathcal{E}}_{\tau}^{\prime}: 0 \rightarrow T \Theta_{m,((1,0), \tau)} \rightarrow T A_{\tau} \rightarrow N^{\prime} \rightarrow 0
$$

and

$$
\overline{\mathcal{E}}_{m \tau}: 0 \rightarrow T \Theta_{m \tau} \rightarrow T A_{m \tau} \rightarrow N \rightarrow 0
$$

be the exact sequences of Hermitian vector bundles whose metrics are $\left(\left.g_{\tau}\right|_{\Theta_{m,((1,0), \tau)}}, g_{\tau}, g_{N^{\prime}}\right)$ and $\left(\left.g_{m \tau}\right|_{\Theta_{m \tau}}, g_{m \tau}, g_{N}\right)$ respectively. Since $d \theta_{\underline{0}}(\tau)$ $=\mu_{m}^{*} d \theta(\cdot, m \tau)$, it follows from $(6.2),(6.3)$ and also the formula of BottChern classes $([3, \mathrm{I}$, Theorem 1.29]) that

$$
\mu_{m}^{*}\left(N_{m \tau}, g_{N_{m \tau}}\right)=\left(N_{\tau}^{\prime}, g_{N_{\tau}^{\prime}}\right), \quad \widetilde{\operatorname{Td}}\left(\overline{\mathcal{E}}_{\tau}^{\prime}\right)=\mu_{m}^{*} \widetilde{\operatorname{Td}}\left(\overline{\mathcal{E}}_{m \tau}\right) .
$$

Similarly, it follows from (6.2) and (6.4) that

$$
\begin{aligned}
& \operatorname{Td}^{-1}\left(L_{\tau}^{-m}, h_{L_{\tau}^{-m}}\right)=\mu_{m}^{*} \operatorname{Td}^{-1}\left(L_{m \tau}^{-1}, h_{L_{m \tau}^{-1}}\right), \\
& \log \left\|\theta_{\frac{0}{m}}(\tau)\right\|_{L_{\tau}^{-m}}^{2}=\mu_{m}^{*} \log \|\theta(\cdot, m \tau)\|_{L_{m \tau}^{-1}}^{2}, \\
& \operatorname{Td}^{-1}\left(N^{\prime}, g_{N^{\prime}}\right)=\mu_{m}^{*} \operatorname{Td}^{-1}\left(N, g_{N}\right) .
\end{aligned}
$$

According to the embeddings $i^{\prime}: \Theta_{m,((1,0), \tau)} \hookrightarrow A_{\tau}$ and $i: \Theta_{m \tau} \hookrightarrow A_{m \tau}$, let

$$
\lambda_{\tau}^{\prime}:=\lambda_{\Theta_{m,((1,0), \tau)}} \otimes \lambda_{A_{\tau}}^{-1} \otimes \lambda_{A_{\tau}}\left(L_{\tau}^{-m}\right)
$$

and

$$
\lambda_{m \tau}:=\lambda_{\Theta_{m \tau}} \otimes \lambda_{A_{m \tau}}^{-1} \otimes \lambda_{A_{m \tau}}\left(L_{\tau}^{-1}\right)
$$

be the determinant lines. Let $\sigma^{\prime} \in \lambda_{\tau}^{\prime}$ and $\sigma \in \lambda_{m \tau}$ be their canonical elements. By Theorem 2.4 together with (6.4) and (6.5), we get

$$
\log \left\|\sigma^{\prime}\right\|_{\lambda_{\tau}^{\prime}, Q}^{2}=\operatorname{deg}\left(\mu_{m}\right) \log \|\sigma\|_{\lambda_{m \tau}, Q}^{2}=m^{g} \log \|\sigma\|_{\lambda_{m \tau}, Q}^{2} .
$$

Put

$$
\begin{array}{r}
\theta_{a}^{*}(\tau):=\left(\operatorname{det} \frac{\operatorname{Im} \tau}{2 m}\right)^{-\frac{1}{2}} \frac{\overline{\theta_{a, 0}(m z, m \tau)}}{\exp 2 \pi m^{t} \operatorname{Im} z(\operatorname{Im} \tau)^{-1} \operatorname{Im} z} \\
\cdot\left(\frac{i}{2}\right)^{g} d \bar{z}_{1} \wedge \cdots \wedge d \bar{z}_{g} .
\end{array}
$$

Since

$$
\theta_{a}^{*}(\tau)=C_{\tau, g}, *\left(\theta_{a} d z_{1} \wedge \cdots \wedge d z_{g}\right),
$$

where $C_{\tau, g}$ is a constant, $*$ the Hodge $*$-operator, and

$$
\left\{\theta_{a} d z_{1} \wedge \cdots \wedge d z_{g}\right\}_{a \in B_{m}}
$$


a basis of $H^{0}\left(A_{\tau}, K_{A_{\tau}} \otimes L_{\tau}^{m}\right)$, we find that $\left\{\theta_{a}^{*}(\tau)\right\}_{a \in B_{m}}$ are harmonic representatives of $H^{g}\left(A_{\tau}, L_{m, \tau}^{-1}\right)$. By (3.7), we get

$$
\begin{aligned}
& \left\langle\theta_{a, 0} d z_{1} \wedge \cdots \wedge d z_{g}, \theta_{b}^{*}(\tau)\right\rangle=\delta_{a b}, \\
& \left(\theta_{a}^{*}(\tau), \theta_{b}^{*}(\tau)\right)_{L^{2}}=\left(\operatorname{det} \frac{2}{m} \operatorname{Im} \tau\right)^{-\frac{1}{2}} \delta_{a b},
\end{aligned}
$$

where $\langle\cdot, \cdot\rangle$ is the natural paring between $H^{0}\left(A_{\tau}, K_{A_{\tau}} \otimes L_{\tau}^{m}\right)$ and $H^{g}\left(A_{\tau}, L_{m, \tau}^{-1}\right)$. Since $H^{0}\left(A_{\tau}, \Omega^{g}\left(\log \Theta_{m,((1,0), \tau)}\right)\right)$ and $H^{0}\left(A_{\tau}, K_{A_{\tau}} \otimes L_{\tau}^{m}\right)$ are identified via the map $\otimes \theta_{\frac{0}{m}}$, i.e.,

$$
\otimes \theta_{\frac{a}{m}}: \frac{\theta_{a}}{\theta_{0}} d z_{1} \wedge \cdots \wedge d z_{g} \rightarrow \theta_{a} d z_{1} \wedge \cdots \wedge d z_{g}
$$

(note that $\theta_{\frac{0}{m}}$ is the defining section of $\Theta_{((1,0), \tau)}$ ), it follows from (4.15) and Proposition 4.5 that $\sigma^{\prime}$ and $\sigma$ are represented as follows:

$$
\left(\sigma^{\prime}\right)^{(-1)^{g}}=s_{0} \otimes 1_{A_{\tau}}^{-1} \otimes \sigma_{L_{\tau}^{-m}}, \quad \sigma^{(-1)^{g}}=\sigma_{\Theta_{m \tau}}^{(-1)^{g}} \otimes 1_{A_{m \tau}}^{-1} \otimes \sigma_{L_{m \tau}^{-1}},
$$

where $\sigma_{\Theta}$ is the section as in Proposition 4.5 and

$$
\begin{aligned}
& s_{0}(\tau)=\bigwedge_{a \in B_{m}} \frac{\theta_{a}}{\theta_{0}} d z_{1} \wedge \cdots \wedge d z_{g}, \\
& \sigma_{L_{\tau}^{-m}}=\bigwedge_{a \in B_{m}} \theta_{a}^{*}(\tau), \\
& \sigma_{L_{m}^{-1}}=\theta_{\frac{0}{1}}^{*}(m \tau),
\end{aligned}
$$

which, together with (6.8), yields

$$
\left\|\sigma_{L_{\tau}^{-m}}\right\|_{L^{2}}^{2}=\left(\operatorname{det} \frac{2}{m} \operatorname{Im} \tau\right)^{-\frac{m^{g}}{2}}=m^{g m^{g}}\left\|\sigma_{L_{m \tau}^{-1}}\right\|_{L^{2}}^{2 m^{g}} .
$$

By Proposition 2.1 we obtain

$$
\begin{aligned}
\log \tau\left(A_{\tau}, L_{\tau}^{-m}\right) & =(-1)^{g+1} \frac{m^{g}}{2} \log \frac{m^{g}}{(2 \pi)^{g}}, \\
\log \tau\left(A_{m \tau}, L_{m \tau}^{-1}\right) & =(-1)^{g+1} \frac{1}{2} \log \frac{1}{(2 \pi)^{g}},
\end{aligned}
$$

which, together with (6.11), implies

$$
\left\|\sigma_{L_{\tau}^{-m}}\right\|_{Q}^{2(-1)^{g}}=m^{\frac{(-1)^{g} g m^{g}}{2}}\left\|\sigma_{L_{m \tau}^{-1}}\right\|_{Q}^{2 m^{g}(-1)^{g}} .
$$


Since $\left\|1_{A_{\tau}}\right\|_{Q}^{2}=1$ by Proposition 2.1, it follows from (6.6) and (6.9) that

$$
\begin{aligned}
\log \left\|\sigma^{\prime}\right\|_{\lambda_{\tau}^{\prime}, Q}^{2(-1)^{g}} & =\log \left\|s_{0}\right\|_{Q}^{2(-1)^{g}}+\log \left\|\sigma_{L_{\tau}^{-m}}\right\|_{Q}^{2(-1)^{g}} \\
& =m^{g}\left(\log \left\|\sigma_{\Theta_{m \tau}}\right\|_{Q}^{2}+\log \left\|\sigma_{L_{m \tau}^{-1}}\right\|_{Q}^{2(-1)^{g}}\right) \\
& =m^{g} \log \|\sigma\|_{\lambda_{m \tau}, Q}^{2(-1)^{g}}
\end{aligned}
$$

which, together with (6.13), gives

$$
\begin{aligned}
m^{g} \log \left\|\sigma_{\Theta_{m \tau}}\right\|_{Q}^{2}= & \log \left\|s_{0}\right\|_{Q}^{2(-1)^{g}}+\log \left\|\sigma_{L_{\tau}^{-m}}\right\|_{Q}^{2(-1)^{g}} \\
& -m^{g} \log \left\|\sigma_{L_{m \tau}^{-1}}\right\|_{Q}^{2(-1)^{g}} \\
= & \log \left\|s_{0}\right\|_{Q}^{2(-1)^{g}}+\log m^{\frac{(-1)^{g} m_{m} g}{2}} .
\end{aligned}
$$

So

$$
m^{\frac{(-1)^{g} g m^{g}}{2}}\left\|s_{0}\right\|_{Q}^{2(-1)^{g}}=\left\|\sigma_{\Theta}(m \tau)\right\|_{Q}^{2 m^{g}},
$$

and by Theorems 5.1 and 5.2 we get

$$
\begin{aligned}
& \left\|s_{0}\right\|_{Q}^{2(-1)^{g}}=(\operatorname{det} \operatorname{Im} \tau)^{\frac{(-1)^{g}(g-1) m^{g}}{2(g+1)}}\left|f_{J_{0}}(\tau)\right|^{\frac{(-1)^{g+1} p_{2}}{(g+1) !}}, \\
& \left\|\sigma_{\Theta}(m \tau)\right\|_{Q}^{2}=(\operatorname{det} \operatorname{Im}(m \tau))^{\frac{(-1) g^{g}(g-1)}{2(g+1)}}\left|\Delta_{g}(m \tau)\right|^{\frac{(-1)^{g+1}}{(g+1) !}}
\end{aligned}
$$

which, combined with $(6.16)$, yields

$$
\begin{gathered}
m^{\frac{(-1)^{g} g m^{g}}{2}}(\operatorname{det} \operatorname{Im} \tau)^{\frac{(-1)^{g}(g-1) m^{g}}{2(g+1)}}\left|f_{J_{0}}(\tau)\right|^{\frac{(-1)^{g+1} 2}{(g+1) !}} \\
=\left\{(\operatorname{det} \operatorname{Im}(m \tau))^{\frac{(-1)^{g}(g-1)}{2(g+1)}}\left|\Delta_{g}(m \tau)\right|^{\frac{(-1)^{g+1} 2}{(g+1) !}}\right\}^{m^{g}} \\
=m^{\frac{(-1)^{g} g(g-1) m^{g}}{2(g+1)}}(\operatorname{det} \operatorname{Im}(m \tau))^{\frac{(-1)^{g}(g-1) m^{g}}{2(g+1)}} \\
\cdot\left|\Delta_{g}(m \tau)\right|^{\frac{(-1)^{g+1} m_{m^{g}}}{(g+1) !}} .
\end{gathered}
$$

Eliminating the power $\frac{(-1)^{g+1}}{(g+1) !}$ from $(6.18)$, we obtain

$$
m^{-\frac{g m^{g}}{2}(g+1) !}\left|f_{J_{0}}(\tau)\right|^{2}=m^{-\frac{g(g-1) m^{g}}{2} g !}\left|\Delta_{g}(m \tau)\right|^{2 m^{g}},
$$

and therefore

$$
\begin{aligned}
\left|f_{J_{0}}(\tau)\right|^{2} & =m^{\frac{g(g+1) m^{g}}{2} g !-\frac{g(g-1) m^{g}}{2} g !}\left|\Delta_{g}(m \tau)\right|^{2 m^{g}} \\
& =m^{g \cdot g ! m^{g}}\left|\Delta_{g}(m \tau)\right|^{2 m^{g}}
\end{aligned}
$$


which, together with the normalization condition, yields the assertion.

q.e.d.

Let $\mathcal{M}\left(\mathfrak{S}_{g}\right)$ be the field of meromorphic functions over $\mathfrak{S}_{g}$. Define a polynomial $\widetilde{\Delta}_{g, m}(u, \tau) \in \mathcal{M}\left(\mathfrak{S}_{g}\right)\left[u_{a}\right]_{a \in B_{m}}$ and a meromorphic function $F_{J}(\tau) \in \mathcal{M}\left(\mathfrak{S}_{g}\right)$ by the following formulas:

$$
\begin{aligned}
& \widetilde{\Delta}_{g, m}(u, \tau):=\frac{\Delta_{g, m}(u, \tau)}{m^{\frac{g \cdot g ! m^{g}}{2}} \Delta_{g}(m \tau)^{m^{g}}}=u_{0}^{m^{g}}+\sum_{J \neq J_{0}} F_{J}(\tau) u^{J}, \\
& F_{J}(\tau)=\frac{f_{J}(\tau)}{f_{J_{0}}(\tau)} .
\end{aligned}
$$

Although $f_{J}(\tau)$ is determined up to complex numbers of modulus one, $F_{J}(\tau)$ is uniquely determined. To study the structure of $\widetilde{\Delta}_{g, m}(u, \tau)$, we need the following theorem due to Mumford.

In the sequel, we always assume that $m$ is even and $\geq 4$. Let

$$
\Phi_{m, \tau}: A_{\tau} \ni z \rightarrow\left(\theta_{a}(m z, m \tau)\right)_{a \in B_{m}} \in \mathbb{P}\left(V_{m}\right)
$$

be the embedding associated to the complete linear system $\left|L_{m, \tau}\right|$ as in (4.4). Let $X_{a}\left(a \in B_{m}\right)$ be the homogeneous coordinates of $\mathbb{P}\left(V_{m}\right)$ corresponding to $\theta_{a}$.

Theorem 6.2 ([23, III, Cor.10.13]). The homogeneous ideal defining $\Phi_{m, \tau}\left(A_{\tau}\right)$ in $\mathbb{P}\left(V_{m}\right)$ is generated by the following equations: For any $a, b, a^{\prime}, b^{\prime} \in \frac{1}{m} \mathbb{Z}^{g} / \mathbb{Z}^{g}$ with $a+b \equiv a^{\prime}+b^{\prime} \bmod \mathbb{Z}^{g}$ and any $d \in \frac{1}{m} \mathbb{Z}^{g}$, $c \in \frac{1}{2} \mathbb{Z}^{g} / \mathbb{Z}^{g}$,

$$
\begin{gathered}
\left(\sum_{\eta} s(c, \eta) \theta_{a^{\prime}+d+\eta, 0}(0, m \tau) \theta_{b^{\prime}+d+\eta, 0}(0, m \tau)\right) \\
\cdot\left(\sum_{\eta} s(c, \eta) X_{a+\eta} X_{b+\eta}\right) \\
=\left(\sum_{\eta} s(c, \eta) \theta_{a+d+\eta, 0}(0, m \tau) \theta_{b+d+\eta, 0}(0, m \tau)\right) \\
\cdot\left(\sum_{\eta} s(c, \eta) X_{a^{\prime}+\eta} X_{b^{\prime}+\eta}\right),
\end{gathered}
$$

where $s(c, \eta):=(-1)^{t}(2 c) \cdot(2 \eta)$ and $\eta$ runs over $\frac{1}{2} \mathbb{Z}^{g} / \mathbb{Z}^{g}$. 
Let $k:=\mathbb{Q}\left(\theta_{a, 0}(0, m \tau) \theta_{b, 0}(0, m \tau)\right)_{a, b \in B_{m}}$ be the field of fractions of the ring $\mathbb{Z}\left[\theta_{a, 0}(0, m \tau) \theta_{b, 0}(0, m \tau)\right]_{a, b \in B_{m}}$ which is a proper subfield of $\mathcal{M}\left(\mathfrak{S}_{g}\right)$. Consider the variety $\mathcal{A}_{m}$ in $\mathbb{P}_{k}^{m^{g}}$ defined by the equations of Theorem 6.2. Let $\mathcal{A}_{m}^{\vee}$ be the projective dual variety of $\mathcal{A}_{m}$ in $\mathbb{P}_{k}^{m^{g}}$. Then, $\mathcal{A}_{m}^{\vee}$ is a hypersurface on $\left(\mathbb{P}_{k}^{m^{g}}\right)^{\vee}$. Let $\left(u_{a}\right)_{a \in B_{m}}$ be the coordinates of $\left(\mathbb{P}_{k}^{m^{g}}\right)^{\vee}$ dual to $\left(X_{a}\right)_{a \in B_{m}}$.

Theorem 6.3. $\widetilde{\triangle}_{g, m}(u, \tau) \in k\left[u_{a}\right]_{a \in B_{m}}$ is the unique defining equation of $\mathcal{A}_{m}^{\vee}$ which is monic in the variable $u_{0}$.

Proof. Let $\Psi(u, \tau) \in k\left[u_{a}\right]_{a \in B_{m}}$ be the unique defining equation of $\mathcal{A}_{m}^{\vee}$ which is monic in the variable $u_{0}$. Let $Z$ be a proper subvariety of $\mathfrak{S}_{g}$ such that both $\Psi(u, \tau)$ and $\Delta_{g}(m \tau)$ are regular over $\mathbb{C}^{m^{g}} \times\left(\mathfrak{S}_{g} \backslash Z\right)$. By definition, for $\tau \in \mathfrak{S}_{g} \backslash Z, \Psi(u, \tau)$ is the unique defining equation of the projective dual variety of $\Phi_{m}\left(A_{\tau}\right)$ which is monic in the variable $u_{0}$. Since $\mathcal{D}_{g, m, \tau}$ in $\S 4$ is the projective dual variety of $\Phi_{m}\left(A_{\tau}\right)$, it follows from Theorem 5.1 (3) and Theorem 6.1 that $\widetilde{\Delta}_{g, m}(u, \tau)$ is also a defining equation of this variety which is monic in the variable $u_{0}$. By the uniqueness of such polynomials, we find $\Psi(u, \tau)=\widetilde{\Delta}_{g, m}(u, \tau)$ for any $\tau \in \mathfrak{S}_{g} \backslash Z$. This prove the assertion. q.e.d.

Since the ideal of relations among $\left\{\theta_{a, 0}(0, m \tau) \theta_{b, 0}(0, m \tau)\right\}_{a, b \in B_{m}}$ are known when $m$ is even and $m \geq 6$ ([23, III, Theorem $10.14 \mathrm{~b})]$ ), it is, in principle, possible to write down the explicit formula for $\widetilde{\Delta}_{g, m}(u, \tau)$ in these cases, though it is quite hard in general. In this sense, we know the structure of $\Delta_{g, m}(u, \tau)$ up to that of $\Delta_{g}(\tau)$. In view of the cases of small genus $(g<5)$, we conjecture the following. (A related question is also raised by Mumford ([24, pp.349]).)

Conjecture 6.1. There exists a constant $C_{g}$ such that $C_{g}^{-1} \Delta_{g}(\tau)$ belongs to the ring $\mathbb{Z}\left[\theta_{a, b}(0, \tau) \theta_{c, d}(0, \tau)\right]_{a, b, c, d \in B_{2}}$, and all the Fourier coefficients of $C_{g}^{-1} \Delta_{g}(\tau)$ belong to $\mathbb{Q}$.

As $C_{2} \in \mathbb{Q}\left(\pi, e^{\zeta^{\prime}(-1)}\right)$ (see Theorem 7.2) and $e^{\zeta^{\prime}(-1)}$ comes from the Gillet-Soulé genus ([26, Chap.VIII, 1.2]), it does not seem to be very strange to expect $C_{g} \in \mathbb{Q}\left(\pi, e^{\zeta^{\prime}(-1)}, \cdots, e^{\zeta^{\prime}(1-g)}\right)$ for general $g>1$.

Remark. By Igusa's theorem [15, Chap.V, Theorem 9 and Corollary], considering the case $m=4$, we know that $\Delta_{g}(\tau)$ belongs to the normalization of the ring $R:=\mathbb{C}\left[\theta_{a, 0}(0,4 \tau) \theta_{b, 0}(0,4 \tau)\right]_{a, b \in B_{4}}$. As $R$ is not integrally closed in general, it is not clear even if $\Delta_{g}(\tau) \in R$. (Note that $\theta_{a, 0}(0,4 \tau)\left(a \in B_{4}\right)$ is a $\mathbb{Q}$-linear combination of $\left\{\theta_{a, b}(0, \tau)\right\}_{a, b \in B_{2}}$ by [23, I, Chap.II, Proposition 1.3].) 


\section{An explicit formula for $\Delta_{2,2}(u, \tau)$}

Let $p: \mathbb{A} \rightarrow \mathfrak{S}_{2}$ be the universal family of Abelian surfaces and $\pi: \Theta_{2} \rightarrow \mathbb{P}^{3} \times \mathfrak{S}_{2}$ the family of curves associated to the complete linear system $\left|L_{2}\right|=|2 \Theta|$ over $\mathbb{A}$ as in section 5 . Let $A_{\tau}$ be the Abelian surface and

$$
\begin{aligned}
\Phi_{|2 \Theta|} & : A_{\tau} \ni z \\
\rightarrow\left(\theta_{\frac{1}{2} 000}(2 z, 2 \tau)\right. & : \theta_{\frac{1}{2} \frac{1}{2} 00}(2 z, 2 \tau): \theta_{0 \frac{1}{2} 00}(2 z, 2 \tau) \\
& \left.: \theta_{0000}(2 z, 2 \tau)\right) \in \mathbb{P}^{3}
\end{aligned}
$$

be the morphism associated to the linear system $|2 \Theta|$. Let $w=(x, y, z, t)$ be the coordinates of $\mathbb{C}^{4}$ and $u=\left(u_{0}, u_{1}, u_{2}, u_{3}\right)$ its dual. (As we refer to Hudson's book ([14]), the order of coordinates is different from that in the previous section.) We often identify $\mathbb{C}^{4}$ and its dual. Put

$$
\begin{aligned}
F(w, \tau):= & A(\tau)\left(x^{4}+y^{4}+z^{4}+t^{4}\right) \\
& +B(\tau)\left(x^{2} t^{2}+y^{2} z^{2}\right)+C(\tau)\left(y^{2} t^{2}+z^{2} x^{2}\right) \\
& +D(\tau)\left(z^{2} t^{2}+x^{2} y^{2}\right)+2 E(\tau) x y z t .
\end{aligned}
$$

Then, $K_{\tau}:=\left\{w \in \mathbb{P}^{3} ; F(w, \tau)=0\right\}$ is a Kummer's quartic surface with 16 nodes as its singular set, and $\Phi_{|2 \Theta|}: A_{\tau} \rightarrow K_{\tau}$ coincides with the double covering map $A_{\tau} \rightarrow A_{\tau} / \pm 1$ (cf. [14, §53, §103]) where $A(\tau), B(\tau), C(\tau), D(\tau), E(\tau)$ are modular forms defined by

$$
\begin{aligned}
& A(\tau):=\left(\alpha^{2} \delta^{2}-\beta^{2} \gamma^{2}\right)\left(\beta^{2} \delta^{2}-\gamma^{2} \alpha^{2}\right)\left(\gamma^{2} \delta^{2}-\alpha^{2} \beta^{2}\right), \\
& B(\tau):=\left(\beta^{4}+\gamma^{4}-\alpha^{4}-\delta^{4}\right)\left(\beta^{2} \delta^{2}-\gamma^{2} \alpha^{2}\right)\left(\gamma^{2} \delta^{2}-\alpha^{2} \beta^{2}\right), \\
& C(\tau):=\left(\gamma^{4}+\alpha^{4}-\beta^{4}-\delta^{4}\right)\left(\alpha^{2} \delta^{2}-\beta^{2} \gamma^{2}\right)\left(\gamma^{2} \delta^{2}-\alpha^{2} \beta^{2}\right), \\
& D(\tau):=\left(\alpha^{4}+\beta^{4}-\gamma^{4}-\delta^{4}\right)\left(\alpha^{2} \delta^{2}-\beta^{2} \gamma^{2}\right)\left(\beta^{2} \delta^{2}-\gamma^{2} \alpha^{2}\right), \\
& E(\tau):= \alpha \beta \gamma \delta\left(\delta^{2}+\alpha^{2}-\beta^{2}-\gamma^{2}\right)\left(\delta^{2}+\beta^{2}-\gamma^{2}-\alpha^{2}\right) \\
& \times\left(\delta^{2}+\gamma^{2}-\alpha^{2}-\beta^{2}\right)\left(\alpha^{2}+\beta^{2}-\gamma^{2}-\delta^{2}\right), \\
& \alpha(\tau):=\theta_{\frac{1}{2} 000}(0,2 \tau), \quad \beta(\tau):=\theta_{\frac{1}{2} \frac{1}{2} 00}(0,2 \tau), \\
& \gamma(\tau):=\theta_{0 \frac{1}{2} 00}(0,2 \tau), \quad \delta(\tau):=\theta_{0000}(0,2 \tau) .
\end{aligned}
$$

We remark that our definition of $A(\tau), B(\tau), C(\tau), D(\tau), E(\tau)$ is slightly different from that of Hudson $[14, \S 53]$ because we use a homogeneous polynomial to write the defining equation of Kummer's surface though Hudson uses an inhomogeneous one. 
On $K_{\tau}$ acts the Heisenberg group $H_{2,2} \cong(\mathbb{Z} / 2 \mathbb{Z})^{4}$ generated by the following projective transformations:

$$
\begin{aligned}
& \sigma_{1}:\left(u_{0}, u_{1}, u_{2}, u_{3}\right) \rightarrow\left(u_{2}, u_{3}, u_{0}, u_{1}\right), \\
& \sigma_{2}:\left(u_{0}, u_{1}, u_{2}, u_{3}\right) \rightarrow\left(u_{1}, u_{0}, u_{3}, u_{2}\right), \\
& \sigma_{3}:\left(u_{0}, u_{1}, u_{2}, u_{3}\right) \rightarrow\left(u_{0}, u_{1},-u_{2},-u_{3}\right), \\
& \sigma_{4}:\left(u_{0}, u_{1}, u_{2}, u_{3}\right) \rightarrow\left(u_{0},-u_{1}, u_{2},-u_{3}\right) .
\end{aligned}
$$

For $\sigma \in H_{2,2}$, put

$$
\left(u_{0}^{\sigma}, u_{1}^{\sigma}, u_{2}^{\sigma}, u_{3}^{\sigma}\right):=\sigma \cdot\left(u_{0}, u_{1}, u_{2}, u_{3}\right) .
$$

Since $H_{2,2}$ acts transitively on $\operatorname{Sing} K_{\tau}$, we get

$$
\operatorname{Sing} K_{\tau}=\left\{\left(\alpha(\tau)^{\sigma}: \beta(\tau)^{\sigma}: \gamma(\tau)^{\sigma}: \delta(\tau)^{\sigma}\right)\right\}_{\sigma \in H_{2,2}} .
$$

Put

$$
G(u, \tau):=\prod_{\sigma \in H_{2,2}}\left(\alpha(\tau)^{\sigma} u_{0}+\beta(\tau)^{\sigma} u_{1}+\gamma(\tau)^{\sigma} u_{2}+\delta(\tau)^{\sigma} u_{3}\right)
$$

Theorem 7.1. There exists a constant $C_{2,2}$ independent of $(u, \tau)$ such that

$$
\Delta_{2,2}(u, \tau)=C_{2,2} F(u, \tau)^{2} G(u, \tau)
$$

Proof. Put

$$
H_{u}:=\left\{w \in \mathbb{P}^{3} ; u_{0} x+u_{1} y+u_{2} z+u_{3} t=0\right\},
$$

$C_{u, \tau}:=K_{\tau} \cap H_{u}$ and $\Theta_{u, \tau}:=\Phi_{|2 \Theta|}^{-1}\left(C_{u, \tau}\right)$. By Theorem 5.1, $\Delta_{2,2}(u, \tau)=$ 0 if and only if $\Theta_{u, \tau}$ is singular, and thus $C_{u, \tau}$ is singular. Let $D_{1}$ and $D_{2}$ be the hypersurface of $\mathbb{P}^{3} \times \mathfrak{S}_{2}$ such that $(u, \tau) \in D_{1}$ iff Sing $C_{u, \tau} \in$ $K_{\tau} \backslash \operatorname{Sing} K_{\tau}$ and $(u, \tau) \in D_{2}$ iff $C_{u, \tau}$ passes through Sing $K_{\tau}$. If $(u, \tau)$ is a generic point of $D_{1}$, since $C_{\tau}$ has only one node (which is different from Sing $K_{\tau}$ ), Sing $\Theta_{u, \tau}$ consists of two nodes because $\Phi_{|2 \Theta|}: \Theta_{u, \tau} \rightarrow C_{\tau}$ is an unramified double cover of $C_{u, \tau}$. If $(u, \tau)$ is a generic point of $D_{2}$, $C_{u, \tau}$ has only one node at one of 16 nodes of $K_{\tau}$, and $\Theta_{u, \tau}$ has only one node at some 2 -torsion point of $A_{\tau}$. Thus we get the following equation of divisors:

$$
\left(\Delta_{2,2}\right)_{0}=2 \bar{D}_{1}+\bar{D}_{2}
$$


where $\bar{D}_{1}$ and $\bar{D}_{2}$ are the closures of $D_{1}$ and $D_{2}$. Clearly, $\bar{D}_{2}=(G)_{0}$ by definition. Suppose that $(u, \tau)$ is a generic point of $D_{1}$. Then, $C_{u, \tau}$ has only one node, say $o \notin \operatorname{Sing} K_{\tau}$. Let $(x, y, z)$ be the local coordinates of $\mathbb{P}^{3}$ around $o, \phi(x, y, z)=0$ be the local defining equation of $K_{\tau}$ at $o$, and $\psi(x, y, z)$ that of $H_{u}$. Since $o$ is a smooth point of $K_{\tau}$, we may assume that $\phi(x, y, z)=x$. Then, the local equation of $C_{u, \tau}$ at $o \in K_{\tau}$ is of the form $\psi(0, y, z)=a y^{2}+b y z+c z^{2}+O(3)=0$ because $\left(C_{u, \tau}, o\right)$ is a node. (Here $O(3)$ means the terms of order $\geq 3$.) As $H_{u}$ is also smooth at $o, \partial \psi / \partial x(0) \neq 0$. Thus, there exists some $\lambda \neq 0$ such that $\phi(x, y, z)-\lambda \psi(x, y, z)=O(2)$ which implies that $H_{u}$ is the tangent plane of $K_{\tau}$ at $o$. Therefore, $u$ belongs to the projective dual of $K_{\tau}$. As $K_{\tau}$ is self-dual $([14, \S 96])$, it follows that $\bar{D}_{1} \supset(F)_{0}$ which, together with (7.15), yields the theorem because both $\Delta_{2,2}$ and $F^{2} \cdot G$ have degree 24 in $u$-variables and weight 20 . q.e.d.

\section{Proposition 7.1.}

$$
F((1,0), \tau)^{2} G((1,0), \tau)=\chi_{2}(2 \tau)^{4} .
$$

Proof. For simplicity, put $I(\tau):=F\left((0,1), \frac{\tau}{2}\right)^{2} G\left((0,1), \frac{\tau}{2}\right)$. By Theorem 6.1 and the fact that $\Delta_{2}(\tau)=C_{2} \chi_{2}(\tau)$, there exists a constant $c$ such that $I(\tau)=c \chi_{2}(\tau)^{4}$. Consider the family $\tau(t)=\left(\begin{array}{ll}\tau & t \\ t & \tau\end{array}\right)$ where $\tau \in \mathbb{H}$ and $t \in \mathbb{C}$ is a small number. Put

$$
\theta_{a b}(\tau):=\theta_{\frac{a}{2} \frac{b}{2}}(0, \tau)
$$

Then,

$$
\chi_{1}(\tau)=\theta_{00}(\tau) \theta_{10}(\tau) \theta_{01}(\tau)
$$

by definition. Since

$$
\begin{gathered}
\alpha(\tau(0) / 2)=\gamma(\tau(0) / 2)=\theta_{00}(\tau) \theta_{10}(\tau) \\
\beta(\tau(0) / 2)=\theta_{10}(\tau)^{2}, \\
\delta(\tau(0) / 2)=\theta_{00}(\tau)^{2}, \\
\left.\partial_{t}^{2}\right|_{t=0}\left\{\theta_{\frac{1}{2} \frac{1}{2} 00}(\tau(t)) \theta_{0000}(\tau(t))-\theta_{\frac{1}{2} 000}(\tau(t)) \theta_{0 \frac{1}{2} 00}(\tau(t))\right\} \\
=-\pi^{2} \chi_{1}\left(\frac{\tau}{2}\right)^{4}, \\
\chi_{1}\left(\frac{1}{2} \tau\right)^{2}=2 \chi_{1}(\tau) \theta_{01}(\tau)^{3}, \quad \theta_{00}(\tau)^{4}=\theta_{10}(\tau)^{4}+\theta_{01}(\tau)^{4},
\end{gathered}
$$


we get

$$
\begin{aligned}
&\left.\lim _{t \rightarrow 0} \frac{I(}{} \frac{}{t^{4}}(t)\right) \\
&=\left\{\theta_{00}(\tau)^{2} \theta_{10}(\tau)^{2} \theta_{01}(\tau)^{4}\right\}^{4} \\
& \times\left\{2 \theta_{00}(\tau)^{2} \theta_{10}(\tau)^{2}\right\}^{2}\left\{\theta_{00}(\tau)^{4} \theta_{10}(\tau)^{4}\right\}^{4} \\
& \times\left\{\frac { 1 } { 2 } \partial _ { t } ^ { 2 } | _ { t = 0 } \left\{\theta_{\frac{1}{2} \frac{1}{2} 00}(\tau(t)) \theta_{0000}(\tau(t))\right.\right. \\
&\left.\left.\quad-\theta_{\frac{1}{2} 000}(\tau(t)) \theta_{0 \frac{1}{2} 00}(\tau(t))\right\}\right\}^{2} \\
&= 16 \pi^{4} \chi_{1}(\tau)^{32}
\end{aligned}
$$

Similarly,

$$
\begin{aligned}
\lim _{t \rightarrow 0} \frac{\chi_{2}(\tau(t))^{4}}{t^{4}} & =\chi_{1}(\tau)^{24}\left(\left.\partial_{t}\right|_{t=0} \theta_{\frac{1}{2} \frac{1}{2} \frac{1}{2} \frac{1}{2}}(0, \tau(t))\right)^{4} \\
& =16 \pi^{4} \chi_{1}(\tau)^{32}
\end{aligned}
$$

(See Appendix for the proofs of (7.16), (7.17), and (7.18).) Comparing (7.17) and (7.18) yields the assertion. q.e.d.

Theorem 7.2. Let $\zeta(s)$ be the Riemann zeta function. Then,

$$
\Delta_{2}(\tau)=2^{-22} \pi^{-14} e^{12 \zeta^{\prime}(-1)} \chi_{2}(\tau) .
$$

Proof. Let $\tau(t)$ be the same as in the proof of Proposition 7.1. Let $A_{t}$ be the Abelian surface with period $\left(1_{2}, \tau(t)\right)$ and $\Theta_{t}$ its theta divisor. When $t=0, A_{0}=E_{\tau} \times E_{\tau}$ and $\Theta_{0}=E_{\tau} \times\left\{\frac{1+\tau}{2}\right\}+\left\{\frac{1+\tau}{2}\right\} \times E_{\tau}$ in the sense of divisor on $A_{0}$ where $E_{\tau}$ is the elliptic curve with period $(1, \tau)$. Put $E_{1}:=E_{\tau} \times\left\{\frac{1+\tau}{2}\right\}$ and $E_{2}:=\left\{\frac{1+\tau}{2}\right\} \times E_{\tau}$. Let $S$ be the small disc centered at 0 . Let $\pi: A \rightarrow S$ be the family of Abelian surfaces such that $\pi^{-1}(t)=A_{t}$, and $\pi: \Theta \rightarrow S$ the degenerating family of curves of genus 2 such that $\pi^{-1}(t)=\Theta_{t}$. Let $\sigma_{\Theta}$ be the same section of $\lambda\left(\mathcal{O}_{\Theta}\right)$ over $S$ as in Proposition 4.5. Let $\sigma_{E_{\tau}}:=1 \otimes d z$ be an element of $\lambda\left(E_{\tau}\right)$ under the identification $H^{1}\left(E_{\tau}, \mathcal{O}_{E_{\tau}}\right)^{\vee}=H^{0}\left(E_{\tau}, \Omega_{E_{\tau}}^{1}\right)$. Then, there exists a natural identification $\sigma_{\Theta}(0)=\sigma_{E_{1}} \otimes \sigma_{E_{2}}$. Let $g_{\tau(t)}={ }^{t} d z(\operatorname{Im} \tau(t))^{-1} d \bar{z}$ be the metric of $T A / S$ and $g_{\Theta / S}$ the induced metric on $T \Theta / S$. Let $\|\cdot\|_{Q}$ be the Quillen metric relative to these Kähler metrics. By Bismut's theorem ([1, Théorème 3$])$, we get

$$
\begin{array}{r}
\lim _{t \rightarrow 0}\left\{\log \left\|\sigma_{\Theta}(t)\right\|_{Q}^{2}+\frac{1}{6} \log \|t\|^{2}-\log \left(\left\|\sigma_{E_{1}}\right\|_{Q}^{2} \cdot\left\|\sigma_{E_{2}}\right\|_{Q}^{2}\right)\right\} \\
=-4 \zeta^{\prime}(-1),
\end{array}
$$


where

$$
\|t\|^{2}=\frac{|t|^{2}}{4 \pi^{2}(\operatorname{Im} \tau)^{2}}
$$

(If $z_{i}$ denotes the coordinate of $E_{1}$ centered at $\frac{1+\tau}{2}$, then we know

$$
\pi\left(z_{1}, z_{2}\right)=z_{1} z_{2}+\frac{t}{2 \pi i}+O\left(t^{2}\right)
$$

around $\Sigma:=\operatorname{Sing} \Theta_{0}$ and

$$
g_{\tau(0)}=(\operatorname{Im} \tau)^{-1}\left(\left|d z_{1}\right|^{2}+\left|d z_{2}\right|^{2}\right)
$$

Thus, Bismut's condition that

$$
\bigwedge^{2}\left(N_{\Sigma / A}^{*}\right) \otimes p_{\Sigma}^{*} \kappa \cong \pi^{*}[0]
$$

is an isometry is equivalent to (7.20) and $\left\|\left.d^{2} \pi\right|_{\Sigma}\right\|=1$.) Since

$$
\begin{aligned}
& \log \left\|\sigma_{\Theta}(t)\right\|_{Q}^{2}=\frac{1}{6} \log \operatorname{det} \operatorname{Im} \tau(t)-\frac{1}{6} \log \left|C_{2} \chi_{2}(\tau(t))\right|^{2}, \\
& \log \left\|\sigma_{E_{i}}\right\|_{Q}^{2}=-\frac{1}{6} \log \left|C_{1} \Delta(\tau)\right|,
\end{aligned}
$$

and $\chi_{1}(\tau)=2 \Delta(\tau)^{\frac{1}{8}}$, it follows from $(7.18-21)$ that

$$
\begin{aligned}
\lim _{t \rightarrow 0} \frac{\left\|\sigma_{\Theta}(t)\right\|_{Q}^{2}\|t\|^{\frac{1}{3}}}{\left\|\sigma_{E_{1}}\right\|_{Q}^{2}\left\|\sigma_{E_{2}}\right\|_{Q}^{2}} \\
\quad=\lim _{t \rightarrow 0} \frac{(\operatorname{det} \operatorname{Im} \tau(t))^{\frac{1}{6}}}{\left|C_{2} \chi_{2}(\tau(t))\right|^{\frac{1}{3}}} \cdot \frac{|t|^{\frac{1}{3}}}{(2 \pi)^{\frac{1}{3}}(\operatorname{Im} \tau)^{\frac{1}{3}}} \cdot\left|C_{1} \Delta(\tau)\right|^{\frac{1}{3}} \\
\quad=\frac{C_{1}^{\frac{1}{3}}}{C_{2}^{\frac{1}{3}}(2 \pi)^{\frac{1}{3}}} \lim _{t \rightarrow 0}\left|\frac{t \Delta(\tau)}{\chi_{2}(\tau(t))}\right|^{\frac{1}{3}} \\
=\left(\frac{C_{1}}{2 \pi C_{2}}\right)^{\frac{1}{3}}\left|\frac{2^{-8} \chi_{1}(\tau)^{8}}{2 \pi \chi_{1}(\tau)^{8}}\right|^{\frac{1}{3}} \\
=\left(\frac{2^{-8} C_{1}}{(2 \pi)^{2} C_{2}}\right)^{\frac{1}{3}}=e^{-4 \zeta^{\prime}(-1)} .
\end{aligned}
$$

As $C_{1}=(2 \pi)^{-12}$ by (1.4), we get $C_{2}=2^{-8}(2 \pi)^{-14} e^{12 \zeta^{\prime}(-1)}$. q.e.d. 
Corollary 7.1. $C_{2,2}=2^{-80} \pi^{-56} e^{48 \zeta^{\prime}(-1)}$.

Proof. Theorems 6.1, 7.1, 7.2 and Proposition 7.1 lead to

$$
C_{2,2}=\frac{\Delta_{2,2}((1,0), \tau)}{\chi_{2}(2 \tau)^{4}}=2^{8} C_{2}^{4}=2^{-80} \pi^{-56} e^{48 \zeta^{\prime}(-1)}
$$

q.e.d.

\section{Appendix. Proofs of (7.16), (7.17) and (7.18)}

Proof of (7.16). The third formula of (7.16) follows from [8, Chap.4, pp.104, (31)]. Since

$$
\theta_{10}\left(\frac{\tau}{2}\right)^{2}=2 \theta_{00}(\tau) \theta_{10}(\tau), \quad \theta_{00}\left(\frac{\tau}{2}\right) \theta_{01}\left(\frac{\tau}{2}\right)=\theta_{01}(\tau)^{2}
$$

by $[8$, Chap.4, p.104, (24)], we get the second formula. (Note that our notation of theta functions and of [8, Chap.4] are related by $\theta_{00}=\theta_{3}$, $\theta_{10}=\theta_{2}$, and $\theta_{01}=\theta_{4}$.) Let us prove the first formula. For simplicity, we write $\theta_{a_{1} a_{2} b_{1} b_{2}}$ instead of $\theta_{\frac{a_{1}}{2} \frac{a_{2}}{2} \frac{b_{1}}{2} \frac{b_{2}}{2}}\left(a_{i}, b_{i} \in\{0,1\}\right)$. Put $\tau(t)=\left(\begin{array}{cc}\tau & t \\ t & \tau\end{array}\right)$ as in the proof of Proposition 7.1. It follows from definition (cf. (3.2)) that

$$
\begin{aligned}
\theta_{0000}(0, \tau(t))= & \sum_{n_{1}, n_{2} \in \mathbb{Z}} \exp \pi i\left[\tau\left(n_{1}^{2}+n_{2}^{2}\right)+2 t n_{1} n_{2}\right], \\
\theta_{1000}(0, \tau(t))= & \sum_{m_{1}, n_{2} \in \mathbb{Z}} \exp \pi i\left[\tau\left\{\left(m_{1}+\frac{1}{2}\right)^{2}+n_{2}^{2}\right\}+2 t\left(m_{1}+\frac{1}{2}\right) n_{2}\right], \\
\theta_{0100}(0, \tau(t))= & \sum_{n_{1}, m_{2} \in \mathbb{Z}} \exp \pi i\left[\tau\left\{n_{1}^{2}+\left(m_{2}+\frac{1}{2}\right)^{2}\right\}+2 t n_{1}\left(m_{2}+\frac{1}{2}\right)\right], \\
\theta_{1100}(0, \tau(t))= & \sum_{m_{1}, m_{2} \in \mathbb{Z}} \exp \pi i\left[\tau\left\{\left(m_{1}+\frac{1}{2}\right)^{2}+\left(m_{2}+\frac{1}{2}\right)^{2}\right\}\right. \\
& \left.+2 t\left(m_{1}+\frac{1}{2}\right)\left(m_{2}+\frac{1}{2}\right)\right] .
\end{aligned}
$$


Therefore, we get

$$
\begin{aligned}
\theta_{1100} \theta_{0000}=\sum \exp \pi i[ & \left\{\left(m_{1}+\frac{1}{2}\right)^{2}+n_{1}^{2}+\left(m_{2}+\frac{1}{2}\right)^{2}+n_{2}^{2}\right\} \\
+ & \left.2 t\left(m_{1} m_{2}+n_{1} n_{2}+\frac{m_{1}}{2}+\frac{m_{2}}{2}+\frac{1}{4}\right)\right] \\
\theta_{1000} \theta_{0100}=\sum \exp \pi i[ & \left\{\left(m_{1}+\frac{1}{2}\right)^{2}+n_{1}^{2}+\left(m_{2}+\frac{1}{2}\right)^{2}+n_{2}^{2}\right\} \\
+ & \left.+2 t\left(m_{1} n_{2}+n_{1} m_{2}+\frac{n_{1}}{2}+\frac{n_{2}}{2}\right)\right]
\end{aligned}
$$

and

$$
\begin{aligned}
& \left.\partial_{t}^{2}\right|_{t=0} \theta_{1100} \theta_{0000} \\
& =-4 \pi^{2} \sum\left(m_{1} m_{2}+n_{1} n_{2}+\frac{m_{1}}{2}+\frac{m_{2}}{2}+\frac{1}{4}\right)^{2} \\
& \cdot \exp \pi i\left[\tau\left\{\left(m_{1}+\frac{1}{2}\right)^{2}+n_{1}^{2}+\left(m_{2}+\frac{1}{2}\right)^{2}+n_{2}^{2}\right\}\right] \\
& \left.\partial_{t}^{2}\right|_{t=0} \theta_{1000} \theta_{0100} \\
& =-4 \pi^{2} \sum\left(m_{1} n_{2}+n_{1} m_{2}+\frac{n_{1}}{2}+\frac{n_{2}}{2}\right)^{2} \\
& \cdot \exp \pi i\left[\tau\left\{\left(m_{1}+\frac{1}{2}\right)^{2}+n_{1}^{2}+\left(m_{2}+\frac{1}{2}\right)^{2}+n_{2}^{2}\right\}\right]
\end{aligned}
$$

Since

$$
\begin{aligned}
&\left(m_{1} m_{2}+n_{1} n_{2}+\frac{m_{1}}{2}+\frac{m_{2}}{2}+\frac{1}{4}\right)^{2} \\
&-\left(m_{1} n_{2}+n_{1} m_{2}+\frac{n_{1}}{2}+\frac{n_{2}}{2}\right)^{2} \\
&=\left(m_{1}+n_{1}+\frac{1}{2}\right)\left(m_{2}+n_{2}+\frac{1}{2}\right) \\
& \cdot\left(m_{1}-n_{1}+\frac{1}{2}\right)\left(m_{2}-n_{2}+\frac{1}{2}\right)
\end{aligned}
$$


it follows from (A.5) and (A.6) that

$$
\begin{aligned}
& \left.\partial_{t}^{2}\right|_{t=0}\left(\theta_{1100} \theta_{0000}-\theta_{1000} \theta_{0100}\right) \\
& =-4 \pi^{2} \sum\left(m_{1}+n_{1}+\frac{1}{2}\right)\left(m_{2}+n_{2}+\frac{1}{2}\right) \\
& \cdot\left(m_{1}-n_{1}+\frac{1}{2}\right)\left(m_{2}-n_{2}+\frac{1}{2}\right) \\
& \cdot \exp \pi i\left[\tau\left\{\left(m_{1}+\frac{1}{2}\right)^{2}+n_{1}^{2}+\left(m_{2}+\frac{1}{2}\right)^{2}+n_{2}^{2}\right\}\right] \\
& =-4 \pi^{2} \sum\left(m_{1}+n_{1}+\frac{1}{2}\right)\left(m_{2}+n_{2}+\frac{1}{2}\right) \\
& \cdot\left(m_{1}-n_{1}+\frac{1}{2}\right)\left(m_{2}-n_{2}+\frac{1}{2}\right) \\
& \cdot \exp \pi i\left[\frac { \tau } { 2 } \left\{\left(m_{1}+\frac{1}{2}+n_{1}\right)^{2}+\left(m_{1}+\frac{1}{2}-n_{1}\right)^{2}\right.\right. \\
& \left.\left.+\left(m_{2}+\frac{1}{2}+n_{2}\right)^{2}+\left(m_{2}+\frac{1}{2}-n_{2}\right)^{2}\right\}\right] \\
& =-4 \pi^{2}\left[\sum_{m, n \in \mathbb{Z}}\left(m+n+\frac{1}{2}\right)\left(m-n+\frac{1}{2}\right)\right. \\
& \cdot \exp \frac{\pi i \tau}{2}\left\{\left(m+n+\frac{1}{2}\right)^{2}\right. \\
& \left.+\exp \pi i \tau\left\{\left(k+\frac{1}{2}\right)^{2}+\left(l+\frac{1}{2}\right)^{2}\right\}\right]^{2} . \\
& \sum_{k, l \in \mathbb{Z}, k=l(2)}\left(k+\frac{1}{2}\right)\left(l+\frac{1}{2}\right) \\
& \left.\left.=-4 \pi^{2}\left[\begin{array}{l}
2 \\
2
\end{array}\right)^{2}\right\}\right]^{2}
\end{aligned}
$$

Since

$$
\begin{aligned}
& \sum_{k, l \in \mathbb{Z}, k=l(2)}\left(k+\frac{1}{2}\right)\left(l+\frac{1}{2}\right) \exp \pi i \tau\left\{\left(k+\frac{1}{2}\right)^{2}+\left(l+\frac{1}{2}\right)^{2}\right\} \\
& =\frac{1}{2} \sum_{k, l \in \mathbb{Z}, k \equiv l(2)}\left(k+\frac{1}{2}\right)\left(l+\frac{1}{2}\right) \exp \pi i \tau\left\{\left(k+\frac{1}{2}\right)^{2}+\left(l+\frac{1}{2}\right)^{2}\right\}
\end{aligned}
$$




$$
\begin{aligned}
& -\frac{1}{2} \sum_{k, l \in \mathbb{Z}, k \equiv l(2)}\left(-k-1+\frac{1}{2}\right)\left(l+\frac{1}{2}\right) \\
& \cdot \exp \pi i \tau\left\{\left(-k-1+\frac{1}{2}\right)^{2}+\left(l+\frac{1}{2}\right)^{2}\right\} \\
= & \frac{1}{2} \sum_{k, l \in \mathbb{Z}}(-1)^{k+l}\left(k+\frac{1}{2}\right)\left(l+\frac{1}{2}\right) \\
& \cdot \exp \pi i \tau\left\{\left(k+\frac{1}{2}\right)^{2}+\left(l+\frac{1}{2}\right)^{2}\right\} \\
= & \frac{1}{2}\left\{\sum_{n \in \mathbb{Z}}(-1)^{n+1}\left(n+\frac{1}{2}\right) \exp \pi i \tau\left(n+\frac{1}{2}\right)^{2}\right\}^{2} \\
= & \frac{1}{2}\left\{\frac{1}{2 \pi} \theta_{11}^{\prime}(0, \tau)\right\}^{2}=\frac{1}{2} \chi_{1}(\tau)^{2},
\end{aligned}
$$

where we have used [23, I, Chap.I, Prop.13.1] to get the last equality, it follows from (A.8) that

$$
\left.\partial_{t}^{2}\right|_{t=0}\left(\theta_{1100} \theta_{0000}-\theta_{1000} \theta_{0100}\right)=-\pi^{2} \chi_{1}\left(\frac{\tau}{2}\right)^{4} .
$$

q.e.d.

Proof of (7.17). For simplicity, put $\alpha(t):=\alpha\left(\frac{\tau(t)}{2}\right)=\theta_{1000}, \beta(t):=$ $\beta\left(\frac{\tau(t)}{2}\right)=\theta_{1100}, \gamma(t):=\gamma\left(\frac{\tau(t)}{2}\right)=\theta_{0100}$, and $\delta(t):=\delta\left(\frac{\tau(t)}{2}\right)=\theta_{0000}$. Then by (A.2) we obtain that

$$
\begin{array}{ll}
\alpha(0)=\theta_{00}(\tau) \theta_{10}(\tau), & \beta(0)=\theta_{10}(\tau)^{2}, \\
\gamma(0)=\theta_{00}(\tau) \theta_{10}(\tau), & \delta(0)=\theta_{00}(\tau)^{2},
\end{array}
$$

which yield

$$
\begin{aligned}
\alpha^{2}(0) \delta^{2}(0)-\beta^{2}(0) \gamma^{2}(0) & =\theta_{00}(\tau)^{6} \theta_{10}(\tau)^{2}-\theta_{00}(\tau)^{2} \theta_{10}(\tau)^{6} \\
& =\theta_{00}(\tau)^{2} \theta_{10}(\tau)^{2}\left(\theta_{00}(\tau)^{4}-\theta_{10}(\tau)^{4}\right) \\
& =\theta_{00}(\tau)^{2} \theta_{10}(\tau)^{2} \theta_{01}(\tau)^{4} \\
& =\chi_{1}(\tau)^{2} \theta_{01}(\tau)^{2}
\end{aligned}
$$

where we have used the third formula of (7.16) to get the third equality, and similarly

$$
\begin{aligned}
\gamma^{2}(0) \delta^{2}(0)-\beta^{2}(0) \alpha^{2}(0) & =\chi_{1}(\tau)^{2} \theta_{01}(\tau)^{2} \\
\beta(0) \delta(0)+\gamma(0) \alpha(0) & =2 \theta_{00}(\tau)^{2} \theta_{10}(\tau)^{2} \\
\alpha(0) \beta(0) \gamma(0) \delta(0) & =\theta_{00}(\tau)^{4} \theta_{10}(\tau)^{4}
\end{aligned}
$$


Since $I(\tau)=C \Delta_{2}(\tau)^{4}$ by Theorem 6.1 and $\Delta_{2}(\tau)$ vanishes of order one along $N_{2}=\left\{\tau \in \mathfrak{S}_{2} ; \tau_{12}=\tau_{21}=0\right\}$ by Theorem 5.2, we find that $\beta(t) \delta(t)-\gamma(t) \alpha(t)=O\left(t^{2}\right)$ as $t \rightarrow 0$. Since $F((1,0), \tau)=A(\tau)$ by $(7.2)$ and

$$
G\left((1,0), \frac{\tau(0)}{2}\right)=(\alpha(0) \beta(0) \gamma(0) \delta(0))^{4}
$$

by (7.14), it follows from (7.3) and (A.11-16) that

$$
\begin{aligned}
&\left.\lim _{t \rightarrow 0} \frac{I(}{} \frac{}{t^{4}}(t)\right) \\
&=\left\{\alpha(0)^{2} \delta(0)^{2}-\beta(0)^{2} \gamma(0)^{2}\right\}^{2} \\
& \cdot\left\{\gamma(0)^{2} \delta(0)^{2}-\alpha(0)^{2} \beta(0)^{2}\right\}^{2} \\
& \cdot\{\beta(0) \delta(0)+\gamma(0) \alpha(0)\}^{2} \cdot \lim _{t \rightarrow 0} \frac{\{\beta(t) \delta(t)-\gamma(t) \alpha(t)\}^{2}}{t^{4}} \\
& \cdot \alpha(0)^{4} \beta(0)^{4} \gamma(0)^{4} \delta(0)^{4} \\
&=\left\{\chi_{1}(\tau)^{2} \theta_{01}(\tau)^{2}\right\}^{2}\left\{\chi_{1}(\tau)^{2} \theta_{01}(\tau)^{2}\right\}^{2}\left\{2 \theta_{00}(\tau)^{2} \theta_{10}(\tau)^{2}\right\}^{2} \\
& \cdot\left\{\left.\frac{1}{2} \partial_{t}^{2}\right|_{t=0}\left(\theta_{1100} \theta_{0000}-\theta_{1000} \theta_{0100}\right)\right\}^{2} \\
& \cdot\left\{\theta_{00}(\tau)^{4} \theta_{10}(\tau)^{4}\right\}^{4} \\
&=\left(\chi_{1}(\tau)^{2} \theta_{01}(\tau)^{2}\right)^{4}\left(2 \theta_{00}(\tau)^{2} \theta_{10}(\tau)^{2}\right)^{2} \\
& \cdot\left(\frac{\pi^{2}}{2} \chi_{1}\left(\frac{\tau}{2}\right)^{4}\right)^{2}\left(\theta_{00}(\tau)^{4} \theta_{10}(\tau)^{4}\right)^{4} \\
&= \pi^{4} \chi_{1}(\tau)^{8} \theta_{01}(\tau)^{8} \theta_{00}(\tau)^{20} \theta_{10}(\tau)^{20} \chi_{1}\left(\frac{\tau}{2}\right)^{8} \\
&= \pi^{4} \chi_{1}(\tau)^{16} \chi_{1}\left(\frac{\tau}{2}\right)^{8} \theta_{00}(\tau)^{12} \theta_{10}(\tau)^{12} \\
&= 2^{4} \pi^{4} \chi_{1}(\tau)^{32},
\end{aligned}
$$

where we have used the first formula of (7.16) to get the third equality, and the second of (7.16) to get the last one. q.e.d.

Proof of (7.18). As is easily verified, even theta constants of genus 2 consist of the following:

$$
\theta_{0000}, \theta_{1000}, \theta_{0100}, \theta_{0010}, \theta_{0001}, \theta_{1100}, \theta_{0011}, \theta_{1001}, \theta_{0110}, \theta_{1111} \text {. }
$$

Since

$$
\theta_{a_{1} a_{2} b_{1} b_{2}}(0, \tau(0))=\theta_{a_{1} b_{1}}(0, \tau) \theta_{a_{2} b_{2}}(0, \tau)
$$


by (A.19) and the definition of $\chi_{i}(i=1,2)$ we obtain that

$$
\lim _{t \rightarrow 0} \frac{\chi_{2}(\tau(t))}{t}=\left.\chi_{1}(\tau)^{6} \cdot \partial_{t}\right|_{t=0} \theta_{1111}(0, \tau(t)) .
$$

As

$$
\begin{aligned}
& \theta_{1111}(0, \tau(t)) \\
& =\sum_{k, l \in \mathbb{Z}}(-1)^{k+l+1} \exp \pi i\left[\tau\left\{\left(k+\frac{1}{2}\right)^{2}+\left(l+\frac{1}{2}\right)^{2}\right\}\right. \\
& \left.+2 t\left(k+\frac{1}{2}\right)\left(l+\frac{1}{2}\right)\right]
\end{aligned}
$$

by definition (cf. (3.2)), it follows from (A.9) that

$$
\begin{aligned}
& \left.\partial\right|_{t=0} \theta_{1111}(0, \tau(t)) \\
& =-2 \pi i \sum_{k, l \in \mathbb{Z}}(-1)^{k+l}\left(k+\frac{1}{2}\right)\left(l+\frac{1}{2}\right) \\
& \cdot \exp \pi i \tau\left[\left\{\left(k+\frac{1}{2}\right)^{2}+\left(l+\frac{1}{2}\right)^{2}\right\}\right] \\
& =-2 \pi i \chi_{1}(\tau)^{2},
\end{aligned}
$$

which, together with (A.21), yields

$$
\lim _{t \rightarrow 0} \frac{\chi_{2}(\tau(t))}{t}=-2 \pi i \chi_{1}(\tau)^{8}
$$

q.e.d.

\section{References}

[1] J.-M. Bismut, Métriques de Quillen et dégénérescence de variétés kählériennes, C. R. Acad. Sci. Paris 319 (1994) 1287-1291.

[2] J.-M. Bismut \& J.-B. Bost, Fibrés déterminants, métrique de Quillen et dégénérescence des courbes, Acta Math. 165 (1990) 1-103.

[3] J.-M. Bismut, H. Gillet \& C. Soulé, Analytic torsion and holomorphic determinant bundles I, II, III, Comm. Math. Phys. 115 (1988) 49-78, 79-126, 301-351.

[4] J.-M. Bismut \& G. Lebeau, Complex immersions and Quillen metrics, Inst. Hautes Études Sci. Publ. Math. 74 (1991) 1-297. 
[5] J.-B. Bost, Intrinsic heights of stable varieties and Abelian varieties, Duke Math. J. 82 (1996) 21-70.

[6] J.-B. Bost, J.-F. Mestre \& L. Moret-Bailly, Sur le calcul explicite des "classes de Chern" des surfaces arithmétiques de genre 2, Astérisque 183 (1990) 69-105.

[7] R. Bott \& S. S. Chern, Hermitian vector bundles and the equidistribution of the zeros of their holomorphic sections, Acta Math. 114 (1968) 71-112.

[8] J. H. Conway, \& N. J. A. Sloane, Sphere packings lattices and groups, (Third Ed.), Springer, Berlin, 1998.

[9] O. Debarre, Le lieu des variétés abéliennes dont le diviseur thêta est singulier a deux composantes, Ann. Sci. École Norm. Sup. 25 (1992) 687-708.

[10] G. Faltings, Calculus on arithmetic surfaces, Ann. of Math. 119 (1984) 612-649.

[11] Lectures on the arithmetic Riemann-Roch theorem, Ann. of Math. Stud. 127 (1992).

[12] Freitag, E. Siegelshe modulfunktionen, Springer, Berlin, 1983.

[13] H. Grauert \& R. Remmert, Plurisubharmonische funktionen in komplexen Räumen, Math. Z. 65 (1956) 175-194.

[14] R. Hudson, Kummer's quartic surface, Cambridge Univ. Press, Cambridge.

[15] J.-I. Igusa, Theta functions, Springer, Berlin, 1972.

[16] (1982) $531-545$.

[17] J. Jorgenson \& J. Kramer, Towards the arithmetic degree of line bundles on abelian varieties, Manuscripta Math. 96 (1998) 335-370.

[18] Star products of Green currents and automorphic forms, Preprint, 1998.

[19] N. Katz, Pinceaux de Lefschetz: théorème d'existence, Groupe de monodromie en géométrie algébrique, Exposé XVII, Sém. Géométrie Algébique 7,II, Lecture Notes in Math. 340 (1973) 213-253.

[20] G. R. Kempf, Complex abelian varieties and theta functions, Springer, Berlin, 1991.

[21] S. Kobayashi, Differential geometry of complex vector bundles, Iwanami Shoten Publishers, Tokyo, 1987.

[22] H. Maass, Siegel's modular forms and dirichlet series, Lecture Notes in Math. 216 (1971).

[23] D. Mumford, Tata lectures on Theta I,III, No. 97, 1983; Progr. Math. 28 (1991). 
[24] - On the Kodaira dimension of the Siegel modular variety, Lecture Notes in Math. 997 (1983) 348-375.

[25] D. B. Ray \& I. M. Singer, Analytic torsion for complex manifolds, Ann. of Math. 98 (1973) 154-177.

[26] C. Soulé, et al. Lectures on Arakelov Geometry, Cambridge Univ. Press, Cambridge.

[27] K. Ueno, Discriminants of curves of genus 2 and arithmetic surfaces, Algebraic Geometry and Commutative Algebra in Honor of Masayoshi Nagata, 1987, 749 770 .

[28] K.-I. Yoshikawa, Smoothing of isolated hypersurface singularities and Quillen metrics, Asian J. Math. 2 (1998) 325-344.

NAGOYA UNIVERSITY, JAPAN 